\title{
Ruelle-Pollicott resonances for real analytic hyperbolic maps
}

\author{
Frédéric Faure* \\ Nicolas Roy ${ }^{\dagger}$
}

11th November 2017

\begin{abstract}
We study two simple real analytic uniformly hyperbolic dynamical systems: expanding maps on the circle $S^{1}$ and hyperbolic maps on the torus $\mathbb{T}^{2}$. We show that the Ruelle-Pollicott resonances which describe time correlation functions of the chaotic dynamics can be obtained as the eigenvalues of a trace class operator in Hilbert space $L^{2}\left(S^{1}\right)$ or $L^{2}\left(\mathbb{T}^{2}\right)$ respectively. The trace class operator is obtained by conjugation of the Ruelle transfer operator in a similar way quantum resonances are obtained in open quantum systems. We comment this analogy.

PACS numbers: 05.45.-a , 05.45.Ac, 05.45.Pq
\end{abstract}

\section{Contents}

1 Introduction $\quad 2$

2 Statement of the results $\quad 4$

2.1 Expanding map on the circle . . . . . . . . . . . . . . . . 4

2.1.1 Dynamical correlation functions . . . . . . . . . . . . . 6

2.1.2 Finite rank approximation . . . . . . . . . . . . . . 6

2.1.3 Exponential concentration of R.P. resonances near zero . . . . . . . 6

2.1.4 Relation with randomly perturbed operators or noisy models . . . . 7

2.1.5 Trace formula . . . . . . . . . . . . . . . 8

2.2 Hyperbolic map on the torus . . . . . . . . . . . . . . . . . . 8

2.2.1 Exponential concentration of R.P. resonances near zero . . . . . . . 10

*Institut Fourier 100, rue des Maths, BP 74 ,38402 St Martin d'Heres, France.

email: frederic.faure@ujf-grenoble.fr http://www-fourier.ujf-grenoble.fr/ faure

${ }^{\dagger}$ Geometric Analysis group, Institut für Mathematik, Humbold Universität, Berlin. roy@math.huberlin.de 
2.2.2 Relation with randomly perturbed operators . . . . . . . . . . 10

2.2 .3 Trace formula . . . . . . . . . . . . . . . . 11

3 Expanding map on the circle $\quad 11$

3.1 Matrix elements of the operator $\hat{M} \ldots \ldots \ldots$. . . . . . . . . . . . . . . . . . . 11

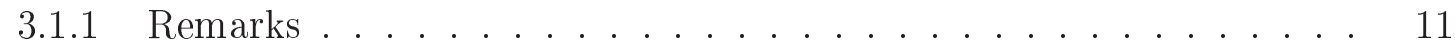

3.1.2 Localization property of matrix elements . . . . . . . . . . . 12

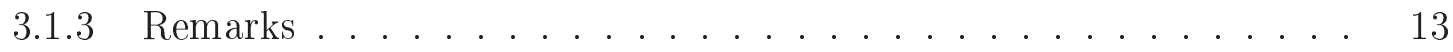

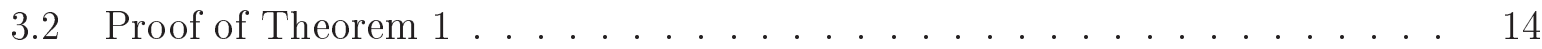

3.2.1 Idea of the proof and remarks . . . . . . . . . . . . . . 14

3.2.2 Exponential decrease of matrix elements . . . . . . . . . . . . . 14

3.2 .3 Trace class operator . . . . . . . . . . . . . . . 15

3.3 Exponential accumulation of R.P. resonances near zero . . . . . . . . . . 16

3.4 Numerical illustrations: Sinai-Ruelle-Bowen Measure and Ruelle-Pollicott

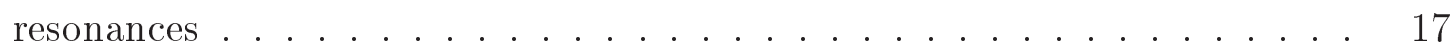

3.4.1 The Sinai-Ruelle-Bowen measure . . . . . . . . . . . . . 17

3.4.2 The Ruelle-Pollicott resonances . . . . . . . . . . . . . . . 18

4 Hyperbolic map on the torus $\quad 19$

4.1 Matrix elements of the operator $\hat{M} \ldots \ldots$. . . . . . . . . . . . 19

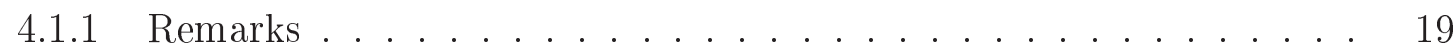

4.1.2 Localization property of the matrix elements . . . . . . . . . . 20

4.2 Proof of Theorem $6 \ldots \ldots \ldots \ldots$. . . . . . . . . . . . . . 21

4.2.1 Proof of the exponential concentration of R.P. resonances . . . . . . 23

5 Conclusion $\quad 24$

\section{Introduction}

In this paper, we study the Ruelle-Pollicott resonances of two simple models which are uniformly hyperbolic: expanding maps on the circle $S^{1}$ and hyperbolic maps on the torus $\mathbb{T}^{2}$. These models are the most simple examples of chaotic dynamical systems, which exhibit strong chaotic properties, such as ergodicity, mixing, decay of correlations, central limit theorem for observables, etc. , see [14][9][3]. Expansivity or hyperbolicity makes every trajectory unstable and are therefore important hypothesis responsible for these chaotic properties. One of these properties, the "decay of time correlation" is fundamental to establish other chaotic properties. Decay of time correlations can be studied by means of the spectral analysis of "Ruelle transfer operators", which transport functions on $S^{1}$ or $\mathbb{T}^{2}$ according to the dynamics [7, 20]. The simplest Ruelle transfer operator is the "pull back operator" or "Koopman operator" defined by: $(\hat{M} \varphi)(x) \stackrel{\text { def }}{=} \varphi(M(x))$, where $M: S^{1} \rightarrow S^{1}$ 
is the map (resp. $M: \mathbb{T}^{2} \rightarrow \mathbb{T}^{2}$ ), and $\varphi \in C^{0}\left(S^{1}\right)$. The time correlation of two functions $\phi, \varphi \in C^{0}\left(S^{1}\right)$ is defined by $C_{\phi, \varphi}(t) \stackrel{\text { def }}{=}\left\langle\phi\left|\hat{M}^{t}\right| \varphi\right\rangle=\int_{S^{1}} \phi(x)\left(\varphi \circ M^{t}(x)\right) d x$. The main effect of the hyperbolic dynamics is to transform an initial function $\varphi$ into a function $\hat{M}^{t} \varphi$ with finer and finer structures, as the time $t$ evolves. In other words, the information on the initial function $\varphi$ is sent towards "microscopic scales", or equivalently at infinity in the Fourier space. On the "macroscopic scale" (i.e., if $\hat{M}^{t} \varphi$ is tested on a regular function $\phi$ ), there remains only a constant function, i.e., the function 1 times a weight $\mu_{S R B}(\varphi)$ where $\mu_{S R B}$ is called the Sinai-Ruelle-Bowen (S.R.B.) measure. The number $\mu_{S R B}(\varphi) \in \mathbb{C}$ is the only information on $\varphi$ which remains on the macroscopic scale after a long time evolution. With this point of view, the decay of time correlation functions $C_{\phi, \varphi}(t)$ is due to the escape of the function $\hat{M}^{t} \varphi$ towards infinity in the Fourier space, implying a decay of the small Fourier components. This suggests to study the decay of correlations using a "window of observation" in the Fourier space, centered on small Fourier components (in the unstable direction). This is the role of the operator $\hat{A}$ below. This situation is very similar to open quantum systems, where the decay of the quantum wave function in a compact domain of space is due to the escape of the wave function towards infinity. In such situations people study the decay by a "complex scaling method" which consists in conjugating the dynamical operator in such a way that the wave function is toned down at infinity ([6], chap. 8). Then, the "quantum resonances" which appear are suitable to describe the decay. Here we will follow this general approach.

We will suppose in both models that the map $M$ is real analytic. We will show that the time correlation functions can be obtained from an "effective dynamical operator" $\hat{R}$ obtained from $\hat{M}$ by a conjugation $\hat{R}=\hat{A} \hat{M} \hat{A}^{-1}$, where $\hat{A}$ tones down the high Fourier modes in the unstable direction of the dynamics. The main result is to show that $\hat{R}$ is a trace class operator in the Hilbert space $L^{2}\left(S^{1}\right)$ (resp. $\left.L^{2}\left(\mathbb{T}^{2}\right)\right)$. The effective long time dynamics is obtained by $\hat{R}^{t}=\hat{A} \hat{M}^{t} \hat{A}^{-1}$ with $t \in \mathbb{N}$, and the spectral properties of $\hat{R}$ are important for that. The eigenvalues of $\hat{R}$ are called the "Ruelle-Pollicott resonances". This approach, with a conjugation, is the one which is usually followed in the complex scaling methods [6]. An alternative but equivalent approach would be to keep the operator $\hat{M}$ but to work with another norm instead of the $L^{2}$ one, namely with $(\phi, \psi)_{A} \stackrel{\text { def }}{=}(\hat{A} \phi, \hat{A} \psi)_{L^{2}}$. This last formulation is preferred in [5][11][15][4]. In this approach, the operator $\hat{A}$ is seen as an isomorphism between two Hilbert spaces, and one gets $(\phi, \hat{M} \psi)_{A}=\left(\phi^{\prime}, \hat{R} \psi^{\prime}\right)_{L^{2}}$ with $\phi^{\prime}=\hat{A} \phi, \psi^{\prime}=\hat{A} \psi$.

The correlation spectrum for analytic maps has already been studied through trace class operators but with different approaches: the case of expanding maps has been studied by D. Ruelle in [20]. The case of analytic hyperbolic maps has been studied by H.H. Rugh in [21]. Our approach which consists in working in the Fourier space (or more technically conjugating $\hat{M}$ by a pseudo-differential operator) has been already investigated recently for hyperbolic diffeomorphisms by V. Baladi and M. Tsujii [4]. In their paper, they consider a much broader class of dynamical systems (they do not suppose analyticity) and they show quasi-compacity for the transfer operator in a Banach space of distributions. Although our 
results are more restrictive, we believe that they have their own interest because of their technical simplicity (we obtain a trace class operator in a Hilbert space and the proof is quite simple). A technical difference between the two approaches appears for example with the choice of the operator $\hat{A}$. We have to choose an operator which has an exponential expression in the Fourier basis, whereas in [4] the operator $\hat{A}$ has an algebraic dependence on the Fourier modes ( $\hat{A}$ is a power of the Laplacian). This difference is crucial to obtain some of the results presented in this paper.

Our techniques do not allows us to treat any hyperbolic maps on the torus, but maps which are closed enough to the linear hyperbolic map ${ }^{1}$. More precisely, as the proof will show in Section 4.2, the technical assumption is that the unstable and stable tangent directions are uniformly contained in constant cones defined by the linear map. This restriction of our method, due to the very simple expression of the operator $\hat{A}$, saves us on the other hand from making a partition of the unity as in [4].

With a different approach, results close to those obtained by V. Baladi et al. were obtained by C. Liverani et al. in [5][11][15]. The connection between dynamical determinants and Ruelle resonances is established there in great generality. See [4] for historical remarks and comparison between the different approaches.

The paper is organized as follows. In Section 2, we define the operator $\hat{R}$ which governs the decay of the correlation function. We state the theorems which say that $\hat{R}$ is a trace class operator in both case $S^{1}$ and $\mathbb{T}^{2}$. These results are proved respectively in Section 3 and 4. Our method is very convenient for numerical analysis since the truncation of the high Fourier modes produces a small error. In Section 3.3, we present numerical illustrations of some aspects of the S.R.B. measure and the R.P. resonances. In Section 2.1.4, we show the equivalence of our approach with a more common approach known as "randomly perturbed dynamics" or "noisy models" [8][5]. We use this equivalence to show the (well known) Trace formula in terms of periodic orbits [3]. We mention that in [11], a powerful method is developed in the $C^{r}$ case and allows the authors to show spectral stability for a wider class of deterministic and random perturbations.

Acknowledgement. We would like to thank Viviane Baladi, Patrick Bernard, Alain Joye, Malik Mezzadri and Stéphane Nonnenmacher for discussions related to this work. FF gratefully acknowledges partial support by "Agence Nationale de la Recherche" under the grant JC05_52556.

\section{Statement of the results}

\subsection{Expanding map on the circle}

Let $M: \mathbb{R} \rightarrow \mathbb{R}$ be the map defined by ${ }^{2}$

$$
M(x)=2 x+f(x)
$$

\footnotetext{
${ }^{1}$ We discuss some possible extension in the conclusion

${ }^{2}$ More generally we could have supposed that $M(x)=d x+f(x)$ with $d \in \mathbb{N}, d \geq 2$. This does not change the results we obtain.
} 
where $f$ is real analytic and periodic: $f(x+1)=f(x), \forall x \in \mathbb{R}$. We suppose moreover that

$$
f_{\min }^{\prime} \stackrel{\text { def }}{=} \min _{x \in \mathbb{R}}\left(\frac{d f}{d x}\right)>-1
$$

so that $M_{x}^{\prime}=2+\frac{d f}{d x}>1$ ( $M$ is called strictly expanding). A simple example used later for numerical illustrations is

$$
f(x)=\frac{\delta}{2 \pi} \sin (2 \pi x), \quad|\delta|<1
$$

For all $n \in \mathbb{N}$ and all $x \in \mathbb{R}, M(x+n) \equiv M(x) \bmod 1$, hence $M$ defines an expanding map on the circle $S^{1}=\mathbb{R} / \mathbb{Z}$ (also denoted by $M$ ). $M$ is not invertible, but it is the simplest model exhibiting chaotic dynamics, see [14]: expansivity is responsible for the high sensitivity to initial conditions, mixing and positive entropy of the dynamics.

The pull-back operator $\hat{M}$ on $L^{2}\left(S^{1}\right)$ is the (non unitary) bounded operator defined $\mathrm{by}^{3}$

$$
(\hat{M} \varphi)(x) \stackrel{\text { def }}{=} \varphi(M x)
$$

For any $n \in \mathbb{Z}$, we denote ${ }^{4}$ by $|n\rangle \in L^{2}\left(S^{1}\right)$ the Fourier mode $\varphi_{n}(x)=\exp (i 2 \pi n x)$, and define the operator $\hat{A}$ by

$$
\hat{A}|n\rangle \stackrel{\text { def }}{=} \exp (-a|n|)|n\rangle, \quad n \in \mathbb{Z}, \quad \text { with } a>0 .
$$

$\hat{A}$ is diagonal in the Fourier basis. The image $C_{A}=\hat{A}\left(L^{2}\left(S^{1}\right)\right)$ is a set of very regular functions (analytic in a complex neighborhood of $S^{1}$ of radius $a$ ). The operator $\hat{A}$ is used to define ${ }^{5}$ the operator:

$$
\hat{R} \stackrel{\text { def }}{=} \hat{A} \hat{M} \hat{A}^{-1}
$$

with domain $C_{A}$, dense in $L^{2}\left(S^{1}\right)$.

In this paper, we will prove the following theorem:

Theorem 1. There exists $a>0$ entering in eq.(3), such that matrix elements of $\hat{R}$ decrease exponentially: $\left|\left\langle n^{\prime}|\hat{R}| n\right\rangle\right|<\exp \left(-c\left(\left|n^{\prime}\right|+|n|\right)\right)$, with $c>0$. In particular $\hat{R}$ extends to a trace class operator in Hilbert space $L^{2}\left(S^{1}\right)$.

${ }^{3}$ We can also consider a more general class of operators, called "Ruelle transfer operator": $\left(\hat{M}_{g} \varphi\right)(x) \stackrel{\text { def }}{=}$ $e^{g(x)} \varphi(M x)$, with complex valued function $g$. The results obtained in this paper extend to this case provided $g$ is real analytic.

${ }^{4}$ Throughout the paper, we use Dirac notations for vectors in Hilbert space $\mathcal{H}=L^{2}\left(S^{1}\right): \varphi \in \mathcal{H}$ is written $|\varphi\rangle$. Its dual metric is written $\langle\varphi|$. A scalar product is written $\langle\phi \mid \varphi\rangle=\int_{S^{1}} \overline{\phi(x)} \varphi(x) d x$. If $\hat{M}$ is an operator, we write $\langle\phi|\hat{M}| \varphi\rangle \stackrel{\text { def }}{=}\langle\phi \mid \hat{M} \varphi\rangle=\left\langle\hat{M}^{*} \phi \mid \varphi\right\rangle$, (where $\hat{M}^{*}$ is the adjoint operator). Finally, $|\phi\rangle\langle\varphi|\stackrel{\text { def }}{=}| \phi\rangle \otimes\langle\varphi|$.

${ }^{5}$ Equivalently $\hat{A}$ can be seen as a change of norm on $L^{2}\left(S^{1}\right)$. 
As the proof will show, the result holds for any $\left.a \in] 0, a_{0}\right]$, with some $a_{0}>0$, and $c$ depends on $a$.

For general results on trace class operators, see [18, 19], or [10] chap. 4. The eigenvalues of $\hat{R}$ are called the Ruelle-Pollicott resonances (R.P.) of the pull back operator $\hat{M}$.

\subsubsection{Dynamical correlation functions}

The operator $\hat{R}$ is useful to study time-correlation functions. Indeed, if $|\phi\rangle \in C_{A}$ is a regular "test function", and $|\varphi\rangle \in L^{2}\left(S^{1}\right)$, then for $t \in \mathbb{N}, C_{\phi, \varphi}(t) \stackrel{\text { def }}{=}\left\langle\phi\left|\hat{M}^{t}\right| \varphi\right\rangle$ can be expressed using $\hat{R}$ as

$$
C_{\phi, \varphi}(t) \stackrel{\text { def }}{=}\left\langle\phi\left|\hat{M}^{t}\right| \varphi\right\rangle=\left(\langle\phi| \hat{A}^{-1}\right) \hat{R}^{t}(\hat{A}|\varphi\rangle)
$$

In "physical terms" it means that the operator $\hat{R}$ is a nice effective operator to express the dynamics of $\hat{M}$ in $L^{2}\left(S^{1}\right)$ provided it is tested on the regular function space $C_{A}$.

\subsubsection{Finite rank approximation}

There is a direct consequence of Theorem 1 which is useful for the numerical computation of Ruelle-Pollicott resonances. Let $\hat{M}_{N}$ be the matrix of the operator $\hat{M}$ expressed in the Fourier basis and truncated to the $N$ first Fourier modes (i.e., $|n| \leq N$, the matrix $\hat{M}_{N}$ has thus size $(2 N+1) \times(2 N+1))$.

Corollary 2. The eigenvalues of $\hat{M}_{N}$ converge towards the Ruelle-Pollicott resonances, when $N \rightarrow \infty$.

Proof. If $\hat{R}_{N}$ is the matrix of the operator $\hat{R}$ restricted to $\mathcal{H}_{N}=\operatorname{Span}\{|n\rangle, \quad|n| \leq N\} \equiv$ $\mathbb{C}^{2 N+1}$, then the eigenvalues of $\hat{R}_{N}$ converge to the R.P. resonances as $N \rightarrow \infty$, because $\hat{R}_{N}$ converges to $\hat{R}$ in operator norm. But in $\mathcal{H}_{N} \equiv \mathbb{C}^{N+1}, \hat{R}_{N}$ is conjugate to $\hat{M}_{N}$ by the invertible and diagonal matrix $\hat{A}_{N}=\operatorname{Diag}(\exp (-a|n|), n=-N \rightarrow N), \hat{R}_{N}$ and $\hat{M}_{N}$ have the same spectrum.

\subsubsection{Exponential concentration of R.P. resonances near zero}

Since $\hat{R}$ is a compact operator (and moreover a trace class operator), its eigenvalues converge to zero. From Theorem 1, matrix elements $\left\langle n^{\prime}|\hat{R}| n\right\rangle$ decrease exponentially fast for large $|n|,\left|n^{\prime}\right|$. A quite direct consequence of this is the exponential concentration of the eigenvalues of $\hat{R}$ near zero:

Theorem 3. Let $\lambda_{i} \in \mathbb{C}, i=0,1, \ldots$ be the non zero eigenvalues of $\hat{R}$ (the RuellePollicott resonances), such that $\left|\lambda_{i+1}\right| \leq\left|\lambda_{i}\right|$, and counting multiplicity. Let $l_{i}=\log \left|\lambda_{i}\right|$ and $C_{1}=\frac{2\left(1+e^{-c}\right)}{\left(1-e^{-c}\right)^{2}}$. For any $i \geq 0$,

$$
l_{i} \leq-\frac{c}{4} i+\log C_{1}
$$


The constant $c$ is given in Theorem 1 and the proof of this theorem is postponed to Section 3.3. (The best estimate is for the largest possible value of $c$, which is neither very explicit in the proof, nor very sharp).

\subsubsection{Relation with randomly perturbed operators or noisy models}

A different approach to obtain Ruelle-Pollicott resonances of transfer operators is to add a small "noise", or "random perturbation" to the dynamical operator $\hat{M}$ at each step of evolution. These models are often used for theoretical or numerical calculations. In [8] Baladi and Young show that for expanding maps, the randomly perturbed operator is compact, and that its eigenvalues are the Ruelle-Pollicott resonances in the limit when the perturbation vanishes. A similar result is shown by Blank Keller and Liverani in [5] for Anosov maps. See also [17]. In this section we consider such "noisy operators" and show with a very simple proof that they have the same eigenvalues as $\hat{R}$ (i.e., the R.P. resonances) when the perturbation vanishes. The same result holds for Anosov maps on $\mathbb{T}^{2}$ considered in the next section.

Let $\Delta \equiv-\frac{d^{2}}{d x^{2}}$ be the Laplacian operator on $S^{1}$, and for $\varepsilon>0$, let

$$
\hat{\mathcal{D}}_{\varepsilon} \stackrel{\text { def }}{=} e^{-\varepsilon \Delta /(2 \pi)^{2}}
$$

be the heat operator. This operator is diagonal in the Fourier basis:

$$
\hat{\mathcal{D}}_{\varepsilon}|n\rangle=e^{-\varepsilon n^{2}}|n\rangle
$$

The main effect of $\hat{\mathcal{D}}_{\varepsilon}$ is to truncate the high Fourier components. In the "real space" $x \in S^{1}$, the operator $\hat{\mathcal{D}}_{\varepsilon}$ convolutes with a Gaussian distribution of size $\sim \sqrt{\varepsilon}$, so $\hat{\mathcal{D}}_{\varepsilon}$ as the same effect as a Gaussian noise.

Let us define the "noisy perturbed operator" by

$$
\hat{M}_{\varepsilon} \stackrel{\text { def }}{=} \hat{M} \hat{\mathcal{D}}_{\varepsilon}
$$

$\hat{M}_{\varepsilon}$ is a Trace class operator because it is the product of $\hat{\mathcal{D}}_{\varepsilon}$ which is Trace class with $\hat{M}$ which is bounded (cf [18] p.207).

Theorem 4. The eigenvalues of the noisy perturbed operator $\hat{M}_{\varepsilon}$ converge to the RuellePollicott resonances, when $\varepsilon \rightarrow 0$.

Proof. Let us define the operator

$$
\hat{R}_{\varepsilon} \stackrel{\text { def }}{=} \hat{R} \hat{\mathcal{D}}_{\varepsilon}=\hat{A} \hat{M} \hat{A}^{-1} \hat{\mathcal{D}}_{\varepsilon}=\hat{A} \hat{M} \hat{\mathcal{D}}_{\varepsilon} \hat{A}^{-1}=\hat{A} \hat{M}_{\varepsilon} \hat{A}^{-1}
$$

where we have used the fact that $\hat{A}$ and $\hat{\mathcal{D}}_{\varepsilon}$ commute. Let $\hat{R}_{\varepsilon, N}$ (resp. $\hat{M}_{\varepsilon, N}$ ) the matrix of the operator $\hat{R}_{\varepsilon}$ (resp. $\hat{M}_{\varepsilon}$ ) expressed in the Fourier basis and truncated to the first $N$ Fourier modes. We have $\hat{R}_{\varepsilon, N}=\hat{A} \hat{M}_{\varepsilon, N} \hat{A}^{-1}$ so the matrices $\hat{R}_{\varepsilon, N}$ and $\hat{M}_{\varepsilon, N}$ have the same spectrum. But $\hat{R}_{\varepsilon, N}$ converges to $\hat{R}_{\varepsilon}$ in operator norm for $N \rightarrow \infty$ (resp. $\hat{M}_{\varepsilon, N} \rightarrow \hat{M}_{\varepsilon}$ for $N \rightarrow \infty)$. We deduce that $\hat{R}_{\varepsilon}$ and $\hat{M}_{\varepsilon}$ have the same spectrum. Now $\hat{R}_{\varepsilon}$ converges to $\hat{R}$ in operator norm for $\varepsilon \rightarrow 0$, which gives that eigenvalues of $\hat{R}_{\varepsilon}$ converge to eigenvalues of $\hat{R}$. 


\subsubsection{Trace formula}

An important feature of Ruelle transfert operators is the existence of exact trace formulas in terms of periodic orbits ([3] p. 100). We just recall here this trace formula for the operator $\hat{R}$ defined by Eq.(4).

Proposition 5. For any $t \geq 1$,

$$
\operatorname{Tr}\left(\hat{R}^{t}\right)=\sum_{x \in F i x\left(M^{t}\right)} \frac{1}{\left|D_{x} M^{t}-1\right|}
$$

where Fix $\left(M^{t}\right)$ denotes the set of fixed points of $M^{t}$, and $D_{x} M^{t}(x)=\frac{d M^{t}}{d x}(x)$.

Proof. We consider first the trace of the operator $\hat{M}_{t, \varepsilon} \stackrel{\text { def }}{=} \hat{M}^{t} \hat{D}_{\varepsilon}$, (similar to Eq.(8)). From ([10] Th. 8.1 p. 70),

$$
\operatorname{Tr}\left(\hat{M}_{t, \varepsilon}\right)=\int_{0}^{1} d x\left\langle x\left|\hat{M}_{t, \varepsilon}\right| x\right\rangle=\int_{0}^{1} d x \delta_{\varepsilon}\left(M^{t}(x)-x\right)
$$

where $\left\langle x^{\prime}\left|\hat{M}_{t, \varepsilon}\right| x\right\rangle$ denotes the Schwartz kernel of the operator $\hat{M}_{t, \varepsilon}$ on $L^{2}\left(S^{1}\right)$ and where $\delta_{\varepsilon}=\hat{D}_{\varepsilon} \delta$ denotes the regularized Dirac distribution at $x=0$ (i.e. $\delta_{\varepsilon}$ is a periodic Gaussian function with width $\sim \sqrt{\varepsilon})$. With the $\left(\left(2^{t}-1\right)\right.$-valued) change of variable $y=M^{t}(x)-x$, we obtain

$$
\operatorname{Tr}\left(\hat{M}_{t, \varepsilon}\right)=\int_{0}^{2^{t}-1} d y \frac{1}{\left|D_{x} M^{t}-1\right|} \delta_{\varepsilon}(y)
$$

so $\operatorname{Tr}\left(\hat{M}_{t, \varepsilon}\right) \rightarrow \sum_{x \in \operatorname{Fix}\left(M^{t}\right)} \frac{1}{\left|D_{x} M^{t}-1\right|}$ for $\varepsilon \rightarrow 0$. On the other hand, with $\hat{R}_{t, \varepsilon} \stackrel{\text { def }}{=} \hat{R}^{t} \hat{\mathcal{D}}_{\varepsilon}$, we show (as in the proof of Theorem 4), that $\operatorname{Tr}\left(\hat{M}_{t, \varepsilon}\right)=\operatorname{Tr}\left(\hat{R}_{t, \varepsilon}\right)$, and that $\operatorname{Tr}\left(\hat{R}_{t, \varepsilon}-\hat{R}^{t}\right) \rightarrow$ 0 , for $\varepsilon \rightarrow 0$. This gives the result.

\subsection{Hyperbolic map on the torus}

With the same approach, one can study a non linear hyperbolic map on the torus (expressed as a linear map with a small perturbation). Let $M_{0} \in S L(2, \mathbb{Z})$ be an hyperbolic matrix (i.e., $\operatorname{Tr} M_{0}>2$ ) and $f: \mathbb{R}^{2} \rightarrow \mathbb{R}^{2}$ be a real analytic periodic function:

$$
f(x+n)=f(x), \forall x \in \mathbb{R}^{2}, \forall n \in \mathbb{Z}^{2}
$$

Let $M: \mathbb{R}^{2} \rightarrow \mathbb{R}^{2}$ be defined to be $M_{0}$ perturbed by $f$ :

$$
M(x)=M_{0}(x)+f(x)
$$

Then $M(x+n) \equiv M(x)\left[\mathbb{Z}^{2}\right]$ hence $M$ induces a map on $\mathbb{T}^{2}$ also denoted by $M$. Structural stability asserts that the map $M$ on $\mathbb{T}^{2}$ is Anosov (uniformly hyperbolic) whenever $\|f\|_{C^{1}}$ is small enough $([2] \text { p.122)([14] p. 89 })^{6}$.

\footnotetext{
${ }^{6}$ In our case $f$ is supposed to be real analytic. So $\|f\|_{C^{1}}$ is controlled by $\|f\|_{C^{0}}$.
} 
The pull-back operator $\hat{M}$ on $L^{2}\left(\mathbb{T}^{2}\right)$ is the bounded operator defined by

$$
(\hat{M} \varphi)(x) \stackrel{\text { def }}{=} \varphi(M x) \text {. }
$$

Note that the operator $\hat{M}$ is not unitary except if $M$ preserves the area on $\mathbb{T}^{2}$.

Example: Choose $M_{0}=\left(\begin{array}{cc}2 & 1 \\ 1 & 1\end{array}\right)$ and

$$
f(x)=\left(0, \frac{\delta}{2 \pi} \sin \left(2 \pi\left(2 x_{1}+x_{2}\right)\right)\right) .
$$

In this example, $M$ preserves $^{7}$ area $d x_{1} d x_{2}$.

For each $n=\left(n_{1}, n_{2}\right) \in \mathbb{Z}^{2}$, denote by $|n\rangle \in L^{2}\left(\mathbb{T}^{2}\right)$ the Fourier mode $\varphi_{n}(x)=$ $\exp (i 2 \pi(n . x))$. Let $u, s \in \mathbb{R}^{2}$ be unstable/stable eigenvectors of the transposed matrix $M_{0}^{\mathfrak{t}}$, i.e., $M_{0}^{\mathfrak{t}} u=e^{\lambda_{0}} u$ and $M_{0}^{\mathfrak{t}} s=e^{-\lambda_{0}} s$, with $\lambda_{0}>0$. A vector $v=\left(v_{1}, v_{2}\right) \in \mathbb{R}^{2}$ is written $\tilde{v} \equiv\left(v_{u}, v_{s}\right)$ with respect to the basis $(u, s)$. In particular $n \in \mathbb{Z}^{2}$ is mapped to $\tilde{n}=\left(n_{u}, n_{s}\right)$. Define the operator $\hat{A}$ by

$$
\hat{A}|n\rangle \stackrel{\text { def }}{=} \exp \left(-a\left|n_{u}\right|+a\left|n_{s}\right|\right)|n\rangle, \quad n \in \mathbb{Z}^{2}, \quad \text { with } a>0 .
$$

It is diagonal in the Fourier basis. $\hat{A}$ is defined on a domain $D_{A} \stackrel{\text { def }}{=} \operatorname{Dom}(\hat{A})=$ $\left\{|\varphi\rangle=\sum_{n} \varphi_{n}|n\rangle\right.$, s.t. $\left.\sum_{n}\left|\varphi_{n}\right|^{2} e^{2 a\left|n_{s}\right|-2 a\left|n_{u}\right|}<\infty, \sum_{n}\left|\varphi_{n}\right|^{2}<\infty\right\}$ dense in $L^{2}\left(\mathbb{T}^{2}\right)$, and consists of functions with exponentially fast decreasing Fourier components (i.e very regular) in the stable direction. Similarly,

$$
\begin{aligned}
C_{A} \stackrel{\text { def }}{=} \operatorname{Dom}\left(\hat{A}^{-1}\right)= & \left\{|\phi\rangle=\sum_{n} \phi_{n}|n\rangle, \text { s.t. } \sum_{n}\left|\phi_{n}\right|^{2} e^{-2 a\left|n_{s}\right|+2 a\left|n_{u}\right|}<\infty, \sum_{n}\left|\phi_{n}\right|^{2}<\infty\right\} \\
& \subset L^{2}\left(\mathbb{T}^{2}\right)
\end{aligned}
$$

consists of functions with exponentially fast decreasing Fourier components in the unstable direction. One checks that $C_{A}=\hat{A}\left(D_{A}\right), D_{A}=\hat{A}^{-1}\left(C_{A}\right)$.

Define

$$
\hat{R} \stackrel{\text { def }}{=} \hat{A} \hat{M} \hat{A}^{-1}
$$

on a suitable domain included in $C_{A}$ (one has $\operatorname{Dom}(\hat{R})=C_{A}$ if $\hat{M}\left(D_{A}\right) \subset D_{A}$ ).

Theorem 6. For a $C^{1}$-small enough perturbation $f$, i.e., $\|f\|_{C^{1}}<\varepsilon$ with $\varepsilon>0$, there exists $a>0$ such that $\hat{R}$ is defined on the domain $\operatorname{Dom}(\hat{R})=C_{A}$ and its matrix elements decrease exponentially: $\left|\left\langle n^{\prime}|\hat{R}| n\right\rangle\right|<\exp \left(-c\left(\left|n_{1}^{\prime}\right|+\left|n_{2}^{\prime}\right|+\left|n_{1}\right|+\left|n_{2}\right|\right)\right)$, with $c>0$. Therefore, $\hat{R}$ extends to a trace class operator in Hilbert space $L^{2}\left(\mathbb{T}^{2}\right)$.

\footnotetext{
${ }^{7}$ Because in this example, $M$ can be written as $M=M_{1} M_{0}$, where $M_{1}$ is an Hamiltonian time 1 flow, generated by Hamiltonian function $H_{1}\left(x_{1}, x_{2}\right)=\frac{\delta}{(2 \pi)^{2}} \cos \left(2 \pi x_{1}\right)$ on $\mathbb{T}^{2}$.
} 
As the proof will show, the result holds for any $\left.a \in] 0, a_{0}\right]$, with some $a_{0}>0$, and $c$ depends on $a$. The operator $\hat{R}$ is useful to study time-correlation functions: if $|\phi\rangle \in C_{A}$ is a regular "test function", and $|\varphi\rangle \in D_{A}$, then

$$
C_{\phi, \varphi}(t) \stackrel{\text { def }}{=}\left\langle\phi\left|\hat{M}^{t}\right| \varphi\right\rangle=\left(\langle\phi| \hat{A}^{-1}\right) \hat{R}^{t}(\hat{A}|\varphi\rangle)
$$

The different corollaries and applications we mentionned for expanding maps, work as well for hyperbolic maps on the torus (with only minor modifications): (1) finite rank approximation, (2) exponential concentration of R.P. resonances near zero, (3) relation with randomly perturbed operators, (4) Trace formula in terms of periodic orbits.

\subsubsection{Exponential concentration of R.P. resonances near zero}

Theorem 7. Let $\lambda_{n} \in \mathbb{C}, n=0,1, \ldots$ be the non zero eigenvalues of $\hat{R}$ (the Ruelle-Pollicott resonances), such that $\left|\lambda_{n+1}\right| \leq\left|\lambda_{n}\right|$, and counting multiplicity. Let $l_{n}=\log \left|\lambda_{n}\right|$. There exists $C_{1}>0$ such that for any $n \geq 0$,

$$
l_{n} \leq-\frac{c}{3} \sqrt{n} \frac{1}{(1+1 / n)}+\log C_{1}
$$

the constant $c$ is given in Theorem 1 , and the proof of the theorem is postponed to Section 4.2.1. The main difference with (6) is the appearance of $n^{1 / 2}$, where the power is $1 / d$ with $d=\operatorname{dim}\left(\mathbb{T}^{2}\right)=2$. Let us remark that such an asymptotic behaviour of eigenvalues is also met in quantum mechanics in the spectrum $\left(E_{n}\right)_{n}$ of the Harmonic Oscillator on $\mathbb{R}^{d}$. From the semi-classical Weyl law $E_{n} \simeq$ Cste $n^{1 / d}$ (see [13] chap. 16). This suggests that (13) could be obtained or interpreted within a semi-classical approach, with a Weyl asymptotic.

\subsubsection{Relation with randomly perturbed operators}

The analysis made in section 2.1.4, can be repeated with no change except for the definition of the heat operator:

$$
\hat{\mathcal{D}}_{\varepsilon}|n\rangle=e^{-\varepsilon\left(n_{1}^{2}+n_{2}^{2}\right)}|n\rangle
$$

which is used to define the randomly perturbed operator:

$$
\hat{M}_{\varepsilon} \stackrel{\text { def }}{=} \hat{M} \hat{\mathcal{D}}_{\varepsilon}
$$

We have

Theorem 8. The eigenvalues of the noisy perturbed operator $\hat{M}_{\varepsilon}$ converge towards the Ruelle-Pollicott resonances, as $\varepsilon \rightarrow 0$.

(The same proof as in section 2.1.4.) 


\subsubsection{Trace formula}

We have the following Trace formula for $\hat{R}^{t}$ in terms of periodic orbits.

Proposition 9. For any $t \geq 1$,

$$
\operatorname{Tr}\left(\hat{R}^{t}\right)=\sum_{x \in \operatorname{Fix}\left(M^{t}\right)} \frac{1}{\left|\operatorname{det}\left(D_{x} M^{t}-I d\right)\right|}
$$

where Fix $\left(M^{t}\right)$ denotes the set of fixed points of $M^{t}$, and $D_{x} M^{t}(x)$ is the differential at point $x \in \mathbb{T}^{2}$.

The proof follows the same lines as for Proposition 5.

\section{Expanding map on the circle}

In this section, we prove Theorem 1.

\subsection{Matrix elements of the operator $\hat{M}$}

Denote $|n\rangle$ the Fourier mode $\varphi_{n}(x)=\exp (i 2 \pi n x)$, with $n \in \mathbb{Z}, x \in S^{1}$. The set $(|n\rangle)_{n \in \mathbb{Z}}$ form an orthonormal basis of $L^{2}\left(S^{1}\right)$ and matrix elements of $\hat{M}$ in this basis are explicitly given by

$$
\left\langle n^{\prime}|\hat{M}| n\right\rangle=\int_{0}^{1} d x \exp \left(i 2 \pi\left(\left(2 n-n^{\prime}\right) x+n f(x)\right)\right)
$$

\subsubsection{Remarks}

- For a vanishing perturbation $f=0$, then

$$
\left\langle n^{\prime}\left|\hat{M}_{0}\right| n\right\rangle=\delta_{2 n=n^{\prime}}
$$

i.e., in the plane $\left(n^{\prime}, n\right)$, matrix elements are zero except on the "line" $n^{\prime}=2 n$. For a non zero perturbation $f$, we will show that matrix elements are very small outside a cone containing this line.

- Since $f$ is real, we have the symmetry

$$
\left\langle-n^{\prime}|\hat{M}|-n\right\rangle=\overline{\left\langle n^{\prime}|\hat{M}| n\right\rangle}
$$

and if $n=0$, we have

$$
\left\langle n^{\prime}|\hat{M}| 0\right\rangle=\delta_{n^{\prime}=0}
$$

Therefore we have only to study matrix elements with $n>0$. 


\subsubsection{Localization property of matrix elements}

For simplicity of the presentation, we borrow notations from semi-classical analysis (see [16]). For $n>0$, let us make the change of variables $\left(n, n^{\prime}\right) \Leftrightarrow(h, p)$, with

$$
h=\frac{1}{n}, \quad p=\frac{1}{n}\left(n^{\prime}-2 n\right)=n^{\prime} h-2
$$

and define $\hbar=h /(2 \pi)$. A matrix element can be written as the oscillating integral:

$$
I_{\hbar}(p) \stackrel{\text { def }}{=}\left\langle n^{\prime}|\hat{M}| n\right\rangle=\int_{0}^{1} d x \exp (i(f(x)-p x) / \hbar)
$$

From the "non stationary phase theorem" below, this integral is "very small" for values of $p$ outside the interval $\left[f_{\min }^{\prime}, f_{\max }^{\prime}\right]$, with

$$
f_{\min }^{\prime} \stackrel{\text { def }}{=} \min _{x} \frac{d f}{d x}, \quad f_{\max }^{\prime} \stackrel{\text { def }}{=} \max _{x} \frac{d f}{d x} .
$$

Theorem 10. "Non stationary phase". Assume that $f(x)$ is a periodic function and can be continued analytically in some strip $|\operatorname{Im}(x)|<Y$. Assume $p /(2 \pi \hbar) \in \mathbb{Z}$, and $I_{\hbar}(p)=\int_{0}^{1} d x \exp (i(f(x)-p x) / \hbar)$. For any $\varepsilon>0$, there exists a constant $C>0$, such that for any $p, \hbar>0$,

$$
\left|I_{\hbar}(p)\right| \leq \min \left(1, e^{-C\left(f_{\min }^{\prime}-p-\varepsilon\right) / \hbar}, e^{-C\left(p-f_{\text {max }}^{\prime}-\varepsilon\right) / \hbar}\right)
$$

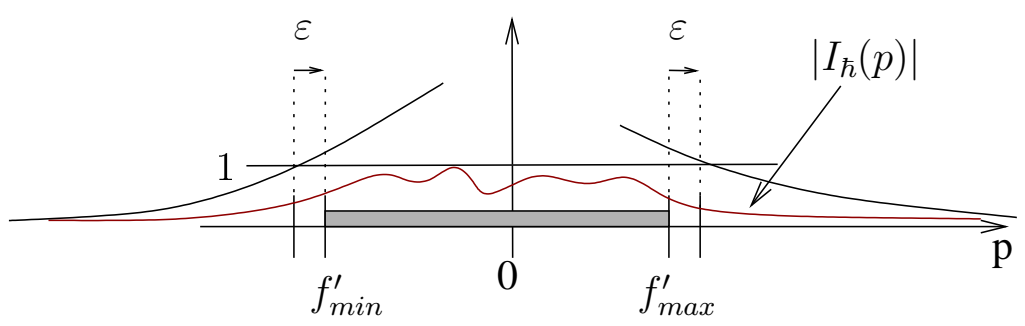

Figure 1: Upper bounds for the function $\left|I_{\hbar}(p)\right|$

Proof. Write $z=x+i y$ and $f(z)=a(z)+i b(z)$, with $a, b$ real valued functions. Analyticity of $f$ gives $\partial_{y} b=\partial_{x} a$. For $y=0$ and $x \in[0,1]$, one has $b(x, 0)=0$ and $\left(\partial_{y} b\right)(x, 0)=$ $\left(\partial_{x} a\right)_{y=0} \geq f_{\min }^{\prime}$. Therefore $\forall \varepsilon>0, \exists y_{0}>0 y_{0}<Y$ such that $b\left(x, y_{0}\right)>\left(f_{\min }^{\prime}-\varepsilon\right) y_{0}$ for all $x$. So for $z=x+i y_{0}$,

$$
|\exp (i(f(z)-p z) / \hbar)|=\exp \left(-\left(b\left(x, y_{0}\right)-p y_{0}\right) / \hbar\right)<\exp \left(-\left(f_{\min }^{\prime}-\varepsilon-p\right) y_{0} / \hbar\right) .
$$

Since $f$ is analytic, we can deform the integration path to $z=x+i y_{0}, x=0 \rightarrow 1, y_{0}$ fixed. This gives

$$
\left|I_{\hbar}(p)\right|<\exp \left(-\left(f_{\min }^{\prime}-\varepsilon-p\right) y_{0} / \hbar\right) .
$$

A similar argument for the integral $\bar{I}_{\hbar}(p)=\int_{0}^{1} d x \exp (i(-f(x)+p x) / \hbar)$ provides

$$
\left|I_{\hbar}(p)\right|<\exp \left(-\left(p-f_{\max }^{\prime}-\varepsilon\right) y_{0}^{\prime} / \hbar\right)
$$

We finally choose $C=\min \left(y_{0}, y_{0}^{\prime}\right)$. 


\subsubsection{Remarks}

- For $p \in\left[f_{\min }^{\prime}, f_{\max }^{\prime}\right]$, the stationary phase formula gives the asymptotic value of $I_{\hbar}(p)$ when $\hbar \rightarrow 0$. It says that the asymptotic value of the matrix element $\left\langle n^{\prime}|\hat{M}| n\right\rangle \equiv$ $I_{\hbar}(p)$ of operator $\hat{M}$ depends only on the point $x^{\prime}$ in the integral, such that $p=\frac{d f}{d x}\left(x^{\prime}\right)$, equivalently $n^{\prime}=\left(2+\frac{d f}{d x}\left(x^{\prime}\right)\right) n$. We comment now on a semi-classical interpretation of this result. The map $M^{-1}$ acting in $S^{1}$ is two valued. Let $\tilde{M}^{-1}$ denotes its lifted action on the cotangent space $T^{*} S^{1}$. If $x=M\left(x^{\prime}\right)=2 x^{\prime}+f\left(x^{\prime}\right)$, then $\frac{\partial}{\partial x^{\prime}}=$ $\left(2+\frac{d f}{d x}\left(x^{\prime}\right)\right) \frac{\partial}{\partial x}$. So, if $(x, k)$ denote coordinates on $T^{*} S^{1}$, and $\left(x^{\prime}, k^{\prime}\right)=\tilde{M}^{-1}(x, k)$, then $x=M\left(x^{\prime}\right)$ and $k^{\prime}=\left(2+\frac{d f}{d x}\left(x^{\prime}\right)\right) k$. So in a sense which need to be precised, $\hat{M}$ acting in $L^{2}\left(S^{1}\right)$ is a "semi-classical quantization" of the map $\tilde{M}^{-1}$ acting in the symplectic space $T^{*} S^{1}$. This "semi-classical aspect" of hyperbolic dynamics will be investigated in a futur work.

- If we come back to variables $n, n^{\prime}$, this last theorem shows that matrix elements $\left\langle n^{\prime}|\hat{M}| n\right\rangle$ in the plane $\left(n^{\prime}, n\right)$ are exponentially small outside the cone defined by (for $n>0)$ :

$$
\left(f_{\min }^{\prime}+2-\varepsilon\right) n<n^{\prime}<\left(f_{\max }^{\prime}+2+\varepsilon\right) n
$$

Expansivity hypothesis of $M$ gives $f_{\min }^{\prime}+2>1$, so for $\varepsilon$ small enough this cone does not contain the diagonal $n^{\prime}=n$. Cf Figure 2 .

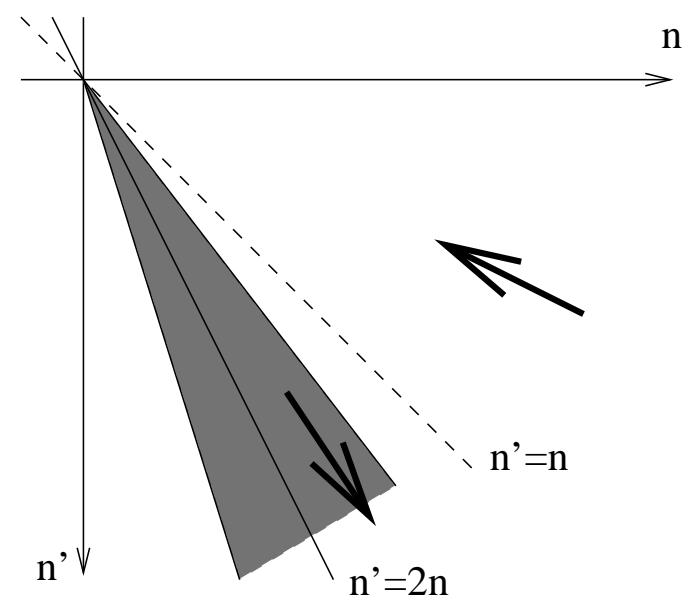

Figure 2: Matrix elements $\left\langle n^{\prime}|\hat{M}| n\right\rangle$ are small outside the grey cone, for $|n| \rightarrow \infty$. For $f=0$, this cone reduces to the line $n^{\prime}=2 n$. These matrix elements can be interpreted as transition amplitudes for the dynamics $n \rightarrow n^{\prime}$. For long times (many iterations), the dynamics goes to infinity in Fourier space on the sector $\left|n^{\prime}\right|>|n|$, and tends to small Fourier components on the sector $\left|n^{\prime}\right|<|n|$ (see the black arrows). Important matrix elements of $\hat{M}$ are localized on the sector $\left|n^{\prime}\right|>|n|$ only, and thus generate an escape towards infinity in Fourier space, responsible for chaos, as discussed in the introduction. 
- With the example (1), we can explicitly express matrix elements in term of Bessel functions of the first kind (see [1] 9.1.21 p. 360):

$$
\left\langle n^{\prime}|\hat{M}| n\right\rangle=(-1)^{\left(2 n-n^{\prime}\right)} J_{\left(2 n-n^{\prime}\right)}(\delta n)
$$

This gives

$$
\left|I_{\hbar}(p)\right|=\left|J_{(-p / h)}\left(\frac{\delta}{h}\right)\right| .
$$

Asymptotic results for Bessel functions (see [1] 9.3.1 and 9.3.2 p. 365) give

$$
\begin{aligned}
\log \left|I_{\hbar}(p)\right| & \sim-\frac{p}{h}\left(\log \left(\frac{2 p}{e \delta}\right)\right), \text { for } h \text { fixed, and } p \rightarrow \infty \\
& \sim-\frac{p}{h}(\alpha-\tanh \alpha), \text { with } \cosh \alpha=\frac{p}{\delta} \geq 1 \text { fixed, and } h \rightarrow 0
\end{aligned}
$$

which are sharper than the upper bound in (17).

\subsection{Proof of Theorem 1}

\subsubsection{Idea of the proof and remarks}

In the linear case, with a vanishing perturbation $f=0$, the result is obvious. Indeed from eq.(15), matrix elements of $\hat{R}$ lie on the line $n^{\prime}=2 n$ and decrease like $\left\langle n^{\prime}|\hat{R}| n\right\rangle=$ $\delta_{n^{\prime}=2 n} e^{a\left(|n|-\left|n^{\prime}\right|\right)}=\delta_{n^{\prime}=2 n} e^{-a|n|}$ (the spectrum is then $\sigma(\hat{R})=\{1\} \cup\{0\}$, with 1 as a simple eigenvalue). Remark that choosing the operator $\hat{A}$ with the algebraic form $\hat{A}|n\rangle=$ $\frac{1}{|n|^{\alpha}}|n\rangle, \quad n \in \mathbb{Z}$ would not give decreasing matrix elements: $\left\langle n^{\prime}|\hat{R}| n\right\rangle=\delta_{n^{\prime}=2 n} \frac{|n|^{a}}{\left|n^{\prime}\right|^{a}}=$ $\delta_{n^{\prime}=2 n} \frac{1}{2^{a}}$. On the other hand, the choice $\hat{A}|n\rangle=e^{-|n|^{a}}|n\rangle$ with $0<a<1$ or $\hat{A}|n\rangle=$ $e^{-a \log ^{2}(1+|n|)}|n\rangle$ would be suitable as well.

In the non linear case, with $f \neq 0$, we have shown that $\left|\left\langle n^{\prime}|\hat{M}| n\right\rangle\right|$ decreases fast outside a cone in the $\left(n^{\prime}, n\right)$ plane. The conjugation with $\hat{A}$ gives the multiplicative factor $e^{+a\left(|n|-\left|n^{\prime}\right|\right)}$, which decreases in the sector $\left|n^{\prime}\right|>|n|$, but increases in the sector $\left|n^{\prime}\right|<|n|$. The expansivity hypothesis insures that the cone is strictly included in the first sector. Moreover, provided $a>0$ is small enough, the decrease of $\left|\left\langle n^{\prime}|\hat{M}| n\right\rangle\right|$ dominates the increase of $e^{+a\left(|n|-\left|n^{\prime}\right|\right)}$ in the sector $\left|n^{\prime}\right|<|n|$. At final result we obtain that $\left|\left\langle n^{\prime}|\hat{R}| n\right\rangle\right|$ decreases exponentially fast for $|n|,\left|n^{\prime}\right| \rightarrow \infty$.

\subsubsection{Exponential decrease of matrix elements}

From (3) and (4) it follows that for any $n>0$ :

$$
\left\langle n^{\prime}|\hat{R}| n\right\rangle=e^{+a\left(n-\left|n^{\prime}\right|\right)}\left\langle n^{\prime}|\hat{M}| n\right\rangle .
$$


Instead of $n^{\prime}, n \in \mathbb{Z}$, we prefer to use "re-normalized" indices for $n>0$, defined by

$$
h=\frac{1}{n}, \quad \nu^{\prime}=n^{\prime} h=p+2
$$

where $h, p$ were already defined in (16).

We can write $e^{+a\left(n-\left|n^{\prime}\right|\right)}=e^{\frac{1}{h} A\left(\nu^{\prime}\right)}$, with $A\left(\nu^{\prime}\right) \stackrel{\text { def }}{=} a\left(1-\left|\nu^{\prime}\right|\right)$, and Equation (17) gives the upper bound:

$$
\left|\left\langle n^{\prime}|\hat{M}| n\right\rangle\right|<e^{\frac{1}{h} B\left(\nu^{\prime}\right)}, \quad B\left(\nu^{\prime}\right) \stackrel{\text { def }}{=} \min \left(0,2 \pi C\left(\nu^{\prime}-b_{\min }\right), 2 \pi C\left(b_{\max }-\nu^{\prime}\right)\right)
$$

with

$$
b_{\min }=2+f_{\min }^{\prime}-\varepsilon, \quad b_{\max }=2+f_{\max }^{\prime}+\varepsilon .
$$

Because $M$ is expanding, we can choose $\varepsilon>0$ such that $\min _{x}\left(M_{x}^{\prime}\right)=2+f_{\min }^{\prime}>1+\varepsilon$, hence $b_{m}>1$. For large $\left|\nu^{\prime}\right|$, the functions $A\left(\nu^{\prime}\right)$ and $B\left(\nu^{\prime}\right)$ and have respective slope $a$ and $2 \pi C$. Choosing $a<2 \pi C$ implies that the maximum of $F\left(\nu^{\prime}\right)=A\left(\nu^{\prime}\right)+B\left(\nu^{\prime}\right)$ is reached for $\nu^{\prime}=b_{m}$. See Figure 3 .

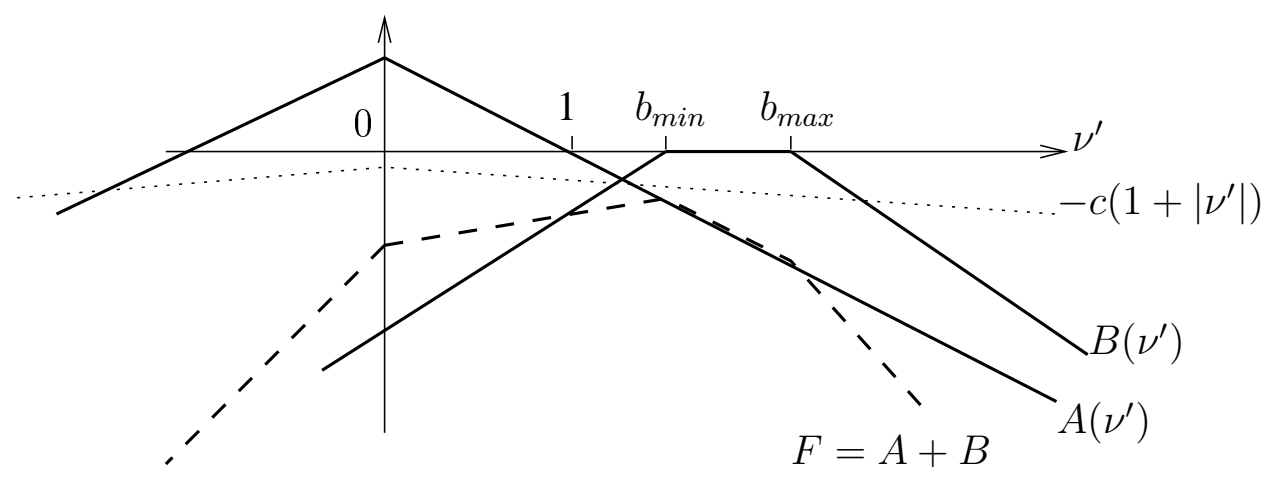

Figure 3: Representation of functions $F\left(\nu^{\prime}\right)=A\left(\nu^{\prime}\right)+B\left(\nu^{\prime}\right)$ for the upper bounds $\left|\left\langle n^{\prime}|\hat{R}| n\right\rangle\right|<\exp \left(\frac{1}{h} F\left(\nu^{\prime}\right)\right)<\exp \left(-\frac{c}{h}\left(1+\left|\nu^{\prime}\right|\right)\right)$.

We need an upper bound $\left|\left\langle n^{\prime}|\hat{R}| n\right\rangle\right|<\exp \left(-c\left(|n|+\left|n^{\prime}\right|\right)\right)=\exp \left(-\frac{c}{h}\left(1+\left|\nu^{\prime}\right|\right)\right)$, therefore we look now for a constant $c>0$ such that $F\left(\nu^{\prime}\right)=A\left(\nu^{\prime}\right)+B\left(\nu^{\prime}\right)<-c\left(1+\left|\nu^{\prime}\right|\right)$, for any $\nu^{\prime} \in \mathbb{R}$. This requires $c<a$ and $F\left(b_{\min }\right) \leq-c\left(1+b_{\min }\right) \Leftrightarrow c<a \frac{b_{\min }-1}{b_{\min }+1}<a$. Consequently we choose $c<a \frac{b_{\min }-1}{b_{\min }+1}$, and this proves the exponential estimates of Theorem 1.

\subsubsection{Trace class operator}

First from $\left|\left\langle n^{\prime}|\hat{R}| n\right\rangle\right|<\exp \left(-c\left(|n|+\left|n^{\prime}\right|\right)\right), \hat{R}$ is a Hilbert-Schmidt operator, therefore bounded. Its domain $C_{A}$ is dense in $L^{2}\left(S^{1}\right)$. From a classical result, $\hat{R}$ extend in a unique way to a bounded operator in $L^{2}\left(S^{1}\right)$. Now let $\hat{B}$ be the operator diagonal in the Fourier 
basis, defined by $\hat{B}|n\rangle=\frac{1}{|n|^{\alpha}}|n\rangle$, with $\alpha>1 / 2 . \quad \hat{B}$ is a Hilbert-Schmidt operator and $\hat{C} \stackrel{\text { def }}{=} \hat{R} \hat{B}^{-1}$ is also an Hilbert-Schmidt operator, so $\hat{R}=\hat{C} \hat{B}$ being a product of two Hilbert Schmidt operators is a trace class operator (cf. [10], Lemma 7.2, p.67).

\subsection{Exponential accumulation of R.P. resonances near zero}

In this section, we prove Theorem 3.

Let $\hat{R}$ be the trace class operator obtained in Theorem 1, with the estimation $\left|\left\langle n^{\prime}|\hat{R}| n\right\rangle\right|<$ $\exp \left(-c\left(|n|+\left|n^{\prime}\right|\right)\right)$ on its matrix elements, with $c>0$. We first deduce an estimation on the singular values of $\hat{R}$ :

Lemma 1. Let $\mu_{j}, j=0,1, \ldots$ be the non zero singular values of $\hat{R}$ (namely the eigenvalues of the self-adjoint operator $\sqrt{\hat{R}^{*} \hat{R}}$ ), such that $\mu_{j+1} \leq \mu_{j}$, repeated as many times as the value of their multiplicity. Then

$$
\mu_{j} \leq C_{1} e^{-c j / 2}
$$

with $C_{1}=\frac{2\left(1+e^{-c}\right)}{\left(1-e^{-c}\right)^{2}}$.

Proof. We borrow an argument from [12] (in the proof of Prop. 3.2). From the min-max theorem,

$$
\mu_{j}=\min _{V \subset L^{2}\left(S^{1}\right), \operatorname{codim} V=j} \max _{v \in V,\|v\|=1}\|\hat{R} v\| .
$$

Consider the Fourier basis $|n\rangle, n \in \mathbb{Z}$ and $V_{l}=\operatorname{span}(|n\rangle)_{|n|>l}$, hence $\operatorname{codim}_{l}=2 l+1$. If $|v\rangle \in V_{l}$, we compute

$$
\begin{aligned}
\| \hat{R}|v\rangle \mid \| & =\| \sum_{n^{\prime} \in \mathbb{Z},|n|>l}\left|n^{\prime}\right\rangle\left\langle n^{\prime}|\hat{R}| n\right\rangle\langle n \mid v\rangle \| \leq \sum_{n^{\prime} \in \mathbb{Z},|n|>l}\left|\left\langle n^{\prime}|\hat{R}| n\right\rangle\langle n \mid v\rangle\right| \\
& \leq\|v\| \sum_{n^{\prime} \in \mathbb{Z},|n|>l} \exp \left(-c\left(|n|+\left|n^{\prime}\right|\right)\right)=\|v\|\left(\sum_{|n|>l} e^{-c|n|}\right) \sum_{n^{\prime} \in \mathbb{Z},} e^{-c\left|n^{\prime}\right|} \\
& =\|v\| 2 S_{l+1}\left(1+2 S_{1}\right)=\|v\| e^{-c(l+1)} 2 S_{0}\left(1+2 S_{1}\right)
\end{aligned}
$$

with $S_{j} \stackrel{\text { def }}{=}=\sum_{n \geq j} e^{-c n}=\frac{e^{-c j}}{1-e^{-c}}=e^{-c j} S_{0}$. We deduce that $\mu_{2 l+1} \leq e^{-c(l+1)} 2 S_{0}\left(1+2 S_{1}\right)$, hence for $j$ odd, $\mu_{j} \leq e^{-c j / 2} e^{-c / 2} 2 S_{0}\left(1+2 S_{1}\right)<C_{1} e^{-c j / 2}$ with $C_{1}=2 S_{0}\left(1+2 S_{1}\right)=$ $2\left(1+e^{-c}\right) /\left(1-e^{-c}\right)^{2}$. For $j$ even, $\mu_{j} \leq \mu_{j-1} \leq e^{-c j / 2} 2 S_{0}\left(1+2 S_{1}\right)=C_{1} e^{-c j / 2}$.

There is a fundamental relation between eigenvalues $\left(\lambda_{j}\right)_{j}$ of $\hat{R}$ (sorted such that $\left|\lambda_{j+1}\right| \leq\left|\lambda_{j}\right|$, and repeated as many times as the value of their multiplicity) and singular values $\left(\mu_{j}\right)_{j}$ (cf. [10], Th. 3.1 p.52):

$$
\prod_{j=0}^{n}\left|\lambda_{j}\right| \leq \prod_{j=0}^{n} \mu_{j}, \quad n \geq 0
$$


For non zero eigenvalues, define

$$
l_{j}=\log \left|\lambda_{j}\right|, \quad m_{j}=\log \mu_{j}
$$

(These sequences tend to $-\infty$ as $j \rightarrow \infty$ ). Then the above inequality reads $\sum_{j=0}^{n} l_{j} \leq$ $\sum_{j=0}^{n} m_{j}$. Eq. (18) gives $m_{j} \leq \log C_{1}-c j / 2$. We deduce that $\sum_{j=0}^{n} l_{j} \leq(n+1) \log C_{1}-$ $\frac{c}{2} \frac{n(n+1)}{2}$, hence

$$
\frac{1}{(n+1)} \sum_{j=0}^{n} l_{j} \leq \log C_{1}-\frac{c}{4} n
$$

But $l_{n} \leq l_{j}$ for $j \leq n$, so $l_{n} \leq \frac{1}{(n+1)} \sum_{j=0}^{n} l_{j} \leq \log C_{1}-\frac{c}{4} n$, which proves Theorem 3 .

\subsection{Numerical illustrations: Sinai-Ruelle-Bowen Measure and Ruelle- Pollicott resonances}

In order to illustrate the previous result, we discuss here some well-known aspects of the S.R.B. measure and Ruelle-Pollicott resonances of Example (1), obtained by numerical diagonalization of operator $\hat{R}$ (in the Fourier basis).

\subsubsection{The Sinai-Ruelle-Bowen measure}

The zero Fourier mode (constant function) $\left|v_{0}\right\rangle=|n=0\rangle$ is an eigenvector of $\hat{M}$ (and thus $\hat{R}$ ) with eigenvalue $\lambda_{0}=1$. It is known that expanding maps such as eq.(2) are mixing [14], which implies as we will see that $\lambda_{0}=1$ is an isolated eigenvalue of multiplicity 1 , and all other eigenvalues of $\hat{R}$ are $\left|\lambda_{i}\right|\left\langle 1, i=1,2 \ldots\right.$. Let $\left.\mid w_{0}\right\rangle \in L^{2}\left(S^{1}\right)$ be the dual eigenvector, i.e., $\left\langle v_{0} \mid w_{0}\right\rangle=1$ and $\hat{R}^{*}\left|w_{0}\right\rangle=\left|w_{0}\right\rangle \Leftrightarrow\left\langle w_{0}\right| \hat{R}=\left\langle w_{0}\right|$. So in operator norm,

$$
\hat{R}^{t} \equiv\left|v_{0}\right\rangle\left\langle w_{0}\left|+\mathcal{O}\left(\left|\lambda_{1}\right|^{t}\right), \quad\right| \lambda_{1}\right|<1 .
$$

If $|\varphi\rangle \in L^{2}\left(S^{1}\right)$, and $|\phi\rangle \in C_{A}$, eq.(5) gives exponential decay of correlation for large $t$ :

$$
\begin{array}{rlrl}
C_{\phi, \varphi}(t)=\left\langle\phi\left|\hat{M}^{t}\right| \varphi\right\rangle & =\left\langle\phi\left|\hat{A}^{-1}\right| v_{0}\right\rangle\left\langle w_{0}|\hat{A}| \varphi\right\rangle+\mathcal{O}\left(\left|\lambda_{1}\right|^{t}\right), & \left|\lambda_{1}\right|<1 \\
& =\left\langle\phi \mid v_{0}\right\rangle\left\langle\mu_{S R B} \mid \varphi\right\rangle+\mathcal{O}\left(\left|\lambda_{1}\right|^{t}\right)
\end{array}
$$

where $\left|\mu_{S R B}\right\rangle \stackrel{\text { def }}{=} \hat{A}\left|w_{0}\right\rangle \in C_{A}$ is called the S.R.B. measure. Its density is a regular (real analytic function) on $S^{1}$, cf Figure 4. The physical meaning of the last equation is that for large $t$ the function $\hat{M}^{t}|\varphi\rangle$ behaves (as seen from test functions, i.e., from a macroscopic point of view) like the constant function $\left|v_{0}\right\rangle$ times $\left\langle\mu_{S R B} \mid \varphi\right\rangle$. This is mixing property. An other interpretation of $\mu_{S R B}$ is that for almost all $x_{0} \in S^{1}$,

$$
\left\langle\mu_{S R B}\right| \equiv \lim _{T \rightarrow \infty} \frac{1}{T} \sum_{t=1}^{T}\left\langle\delta_{M^{t} x_{0}}\right|
$$

(equality of measures) where $\delta_{x}$ is the Dirac measure at $x \in S^{1}$. The right hand side is called the "physical measure", because it is constructed from a typical trajectory ([7] p.640, [3] p.73), cf Figure 4. 


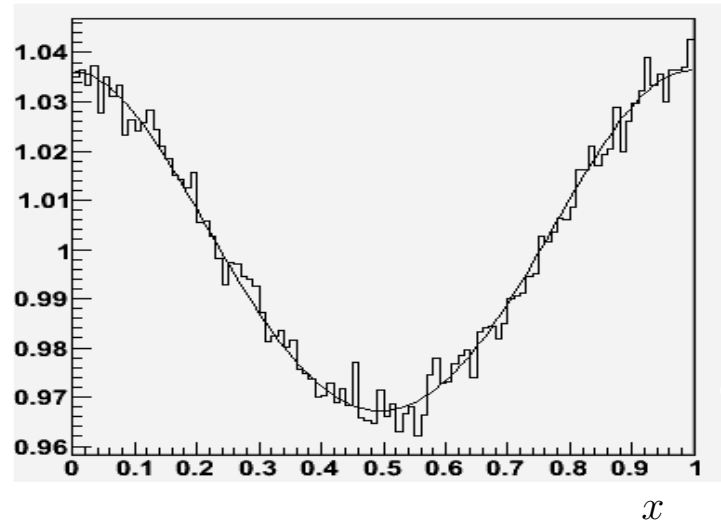

Figure 4: S.R.B. measure: the solid line is the density $\mu_{S R B}(x)$ computed from a numerical diagonalization of $\hat{R}$ (in the Fourier basis), for the perturbation given in (1), with $\delta=0.4$. The histogram is constructed from a trajectory of length $T=10^{7}$ starting from a random initial point $x_{0}$.

\subsubsection{The Ruelle-Pollicott resonances}

Suppose for simplicity that the first $N$ eigenvalues of $\hat{R}$ are simple, $\hat{R}\left|v_{i}\right\rangle=\lambda_{i}\left|v_{i}\right\rangle, i=0 \rightarrow$ $(N-1)$, with $\lambda_{0}=1,\left|\lambda_{i}\right|<1,\left|\lambda_{i+1}\right| \leq\left|\lambda_{i}\right|$, and $\left|\lambda_{N}\right|<\left|\lambda_{N-1}\right|$. Let us write $\left|w_{i}\right\rangle$ the dual vectors ,i.e., $\left\langle w_{i} \mid v_{j}\right\rangle=\delta_{i, j}$ and $\hat{R}^{*}\left|w_{i}\right\rangle=\bar{\lambda}_{i}\left|w_{i}\right\rangle \Leftrightarrow\left\langle w_{i}\right| \hat{R}=\lambda_{i}\left\langle w_{i}\right|$. Then

$$
\hat{R}^{t} \equiv\left|v_{0}\right\rangle\left\langle w_{0}\left|+\sum_{i=1}^{N-1} \lambda_{i}^{t}\right| v_{i}\right\rangle\left\langle w_{i}\left|+\mathcal{O}\left(\left|\lambda_{N}\right|^{t}\right), \quad\right| \lambda_{N}\right|<1
$$

shows that the Ruelle-Pollicott resonances $\lambda_{i}$ govern the asymptotic behaviour of the correlation functions (5) and the convergence towards equilibrium:

$$
C_{\phi, \varphi}(t)=\left\langle\phi\left|\hat{M}^{t}\right| \varphi\right\rangle=\left\langle\phi \mid v_{0}\right\rangle\left\langle\mu_{S R B} \mid \varphi\right\rangle+\sum_{i=1}^{N-1} \lambda_{i}^{t}\left\langle\phi\left|\hat{A}^{-1}\right| v_{i}\right\rangle\left\langle w_{i}|\hat{A}| \varphi\right\rangle+\mathcal{O}\left(\left|\lambda_{N}\right|^{t}\right)
$$

Note that in this last expression $\left|v_{i}\right\rangle,\left|w_{i}\right\rangle \in L^{2}\left(S^{1}\right)$, hence $\hat{A}\left|w_{i}\right\rangle \in C_{A}$ is a regular function, but $\hat{A}^{-1}\left|v_{i}\right\rangle$ may not belong to $L^{2}\left(S^{1}\right)$. We have to interpret $\hat{A}^{-1}\left|v_{i}\right\rangle$ as a linear form on the space $C_{A}$. Vectors $\left|v_{i}\right\rangle,\left|w_{i}\right\rangle$ depend on the choice of operator $\hat{A}$, but eigenvalues $\lambda_{i}$, and distributions $\hat{A}\left|w_{i}\right\rangle, \hat{A}^{-1}\left|v_{i}\right\rangle$ do not.

Let us write $\lambda_{i}=\rho_{i} e^{i \theta_{i}}, \quad \rho_{i}>0$, hence $\log \lambda_{i}=\log \left(\rho_{i}\right)+\mathrm{i} \theta_{i}$. Figure (5) shows the 9 first Ruelle-Pollicott resonances $\log \left(\lambda_{i}\right)$, obtained by a numerical diagonalization of $\hat{R}$ in the Fourier basis. In particular, we remark that there are two symmetric clusters of eigenvalues. $\lambda_{4}$ and $\lambda_{5}$ are very close from each other: $\log \rho_{4}=-5.271, \theta_{4}=1.101$, and $\log \rho_{5}=-5.285, \theta_{4}=1.119$. We have no explanation for this. In semi-classical analysis such clusters occur because of the "tunnelling effect". It would be nice to find such a semiclassical interpretation here. Note that (3) predicts that the values $\log \left(\rho_{i}\right)$ tend at least linearly to $-\infty$, as $i \rightarrow \infty$. 


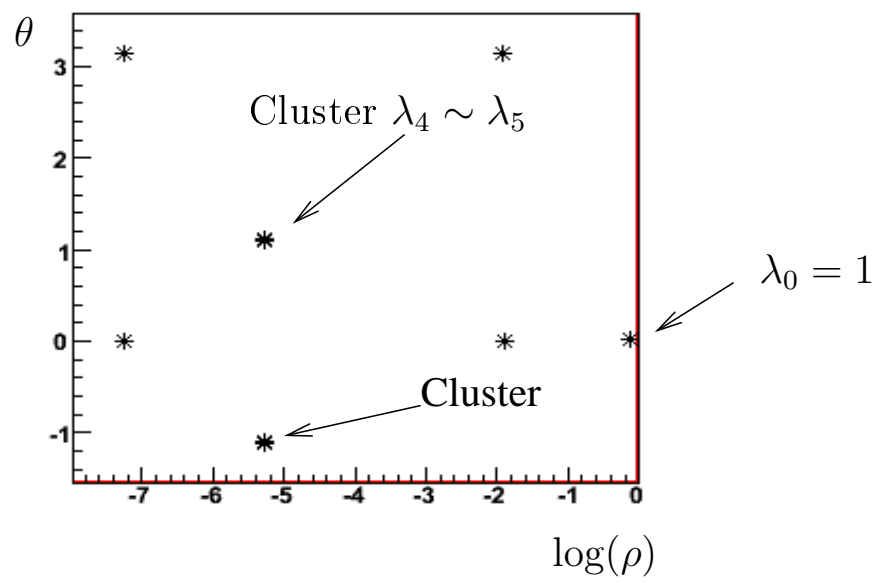

Figure 5: The first nine Ruelle-Pollicott resonances $\lambda_{i}=\rho_{i} e^{i \theta_{i}}$ in log scale, in example Eq.(1), with $\delta=0.4$. We remark two clusters of nearby eigenvalues.

\section{Hyperbolic map on the torus}

We follow essentially the same lines as in the case of an expanding map on the circle, in order to prove Theorem 6 .

\subsection{Matrix elements of the operator $\hat{M}$}

Consider the operator $\hat{M}$ defined in Eq.(9). Let $|n\rangle$ denote the Fourier mode on $\mathbb{T}^{2}=$ $\mathbb{R}^{2} / \mathbb{Z}^{2}$, defined by $\varphi_{n}(x)=\exp (i 2 \pi n \cdot x)$, with $n \in \mathbb{Z}^{2}, x \in \mathbb{T}^{2}$. The set $(|n\rangle)_{n \in \mathbb{Z}^{2}}$ forms a orthonormal basis of $L^{2}\left(\mathbb{T}^{2}\right)$, and matrix elements of $\hat{M}$ in this basis are explicitly given by:

$$
\begin{aligned}
\left\langle n^{\prime}|\hat{M}| n\right\rangle & =\int_{\mathbb{T}^{2}} d x \exp \left(-i 2 \pi n^{\prime} \cdot x\right) \exp \left(i 2 \pi n \cdot\left(M_{0}(x)+f(x)\right)\right) \\
& =\int_{\mathbb{T}^{2}} d x \exp \left(i 2 \pi\left(\left(M_{0}^{\mathfrak{t}}(n)-n^{\prime}\right) \cdot x+n \cdot f(x)\right)\right)
\end{aligned}
$$

with transposed matrix $M_{0}^{\mathrm{t}}$.

\subsubsection{Remarks}

- For a vanishing perturbation $f=0$, then

$$
\left\langle n^{\prime}\left|\hat{M}_{0}\right| n\right\rangle=\delta_{n^{\prime}=M_{0}^{\dagger} n}
$$

For a non vanishing perturbation $f$, we will show now that in the plane $n^{\prime}=\left(n_{1}^{\prime}, n_{2}^{\prime}\right)$ matrix elements are very small outside some domain surrounding the point $n^{\prime}=M_{0}^{\mathrm{t}} n$. 
- Since $f$ is real, we have the symmetry

$$
\left\langle-n^{\prime}|\hat{M}|-n\right\rangle=\overline{\left\langle n^{\prime}|\hat{M}| n\right\rangle}
$$

and if $n=0$, we have

$$
\left\langle n^{\prime}|\hat{M}| 0\right\rangle=\delta_{n^{\prime}=0} .
$$

- With the example given by Eq.(10), one can compute explicitly the matrix elements in terms of the Bessel functions of the first kind (cf [1] 9.1.21 p.360):

$$
\left\langle n^{\prime}|\hat{M}| n\right\rangle=(-1)^{N_{1}} \delta_{N_{2}=0} J_{N_{1}}\left(\delta n_{2}\right) \text {, with } N=\left(n-\left(M_{0}^{-1}\right)^{\mathfrak{t}}\left(n^{\prime}\right)\right)=\left\{\begin{array}{c}
N_{1}=n_{1}-n_{1}^{\prime}+n_{2}^{\prime} \\
N_{2}=n_{2}+n_{1}^{\prime}-2 n_{2}^{\prime}
\end{array}\right.
$$

\subsubsection{Localization property of the matrix elements}

Let us make the following change of variables $\left(n, n^{\prime}\right) \Leftrightarrow(h, \nu, p)$, for $n \neq 0$,

$$
\nu=\frac{n}{|n|} \in S^{1}, \quad h=\frac{1}{|n|}>0, \quad p=h\left(n^{\prime}-M_{0}^{\mathfrak{t}}(n)\right) \in \mathbb{R}^{2},
$$

with $|n|=\sqrt{n_{1}^{2}+n_{2}^{2}}$ and $S^{1}$ the unit circle in Fourier space $\mathbb{R}^{2}$. Define $\hbar=h /(2 \pi)$. Any matrix element can be written as the oscillating integral

$$
I_{\hbar, \nu}(p) \stackrel{\text { def }}{=}\left\langle n^{\prime}|\hat{M}| n\right\rangle=\int_{\mathbb{T}^{2}} d x \exp (i(\nu \cdot f(x)-p \cdot x) / \hbar) .
$$

Theorem 11. "Non stationary phase". Let $f: \mathbb{T}^{2} \rightarrow \mathbb{R}^{2}$ be real analytic, $\hbar>0, \nu \in$ $U(1)$, and $p /(2 \pi \hbar) \in \mathbb{Z}^{2}$. Then $\nu \cdot\left(D_{x} f\right) \in \mathbb{R}^{2}$. Consider the compact domain $\mathcal{E} \stackrel{\text { def }}{=}$ $\left\{\nu \cdot\left(D_{x} f\right)\right.$ s.t. $\left.x \in \mathbb{T}^{2}, \nu \in S^{1}\right\} \subset \mathbb{R}^{2}$ which contains 0 . We denote by $\left[f_{1, \text { min }}^{\prime}, f_{1, \text { max }}^{\prime}\right]$ and $\left[f_{2, \text { min }}^{\prime}, f_{2, \max }^{\prime}\right]$ the projections of $\mathcal{E}$ the axis $p_{1}$ and $p_{2}$ respectively. See Figure 6 . For any $\varepsilon>0$, there exists $C>0$, such that for any $p=\left(p_{1}, p_{2}\right)$ with $p_{1}<f_{1, \text { min }}^{\prime}-\varepsilon$, any $\hbar>0$ and any $\nu \in S^{1}$, one has

$$
\left|I_{\hbar, \nu}(p)\right| \leq e^{-C\left(f_{1, \text { min }}^{\prime}-p_{1}-\varepsilon\right) / \hbar}
$$

Similarly we have exponential upper bounds for the other three half-planes $p_{1}>f_{1, \max }^{\prime}+\varepsilon$, $p_{2}<f_{2, \min }^{\prime}-\varepsilon$ and $p_{2}>f_{2, \max }^{\prime}+\varepsilon$ with the same constant $C$. Moreover we have always the general bound $\left|I_{\hbar, \nu}(p)\right|<1$.

Proof. Let $\varepsilon>0$ and write $I_{\hbar, \nu}(p)=\int_{0}^{1} d x_{2} e^{-i p_{2} x_{2} / \hbar} \mathcal{I}_{1}\left(x_{2}\right)$ with $\mathcal{I}_{1}\left(x_{2}\right)=\int_{0}^{1} d x_{1} e^{i\left(\nu \cdot f\left(x_{1}, x_{2}\right)-p_{1} x_{1}\right) / \hbar}$. For $\nu, x_{2}$ fixed, let $\tilde{f}\left(x_{1}\right)=\nu \cdot f\left(x_{1}, x_{2}\right)$. Then it follows from (17) that,

$$
\left|\mathcal{I}_{1}\left(x_{2}\right)\right|<e^{-C_{1}\left(\tilde{f}_{\min }^{\prime}-p_{1}-\varepsilon\right) / \hbar}
$$

where $C_{1}$ and $\tilde{f}_{\min }^{\prime}$ depend on $\nu$ and $x_{2}$. Let $f_{1, \min }^{\prime}=\min _{\nu, x_{2}}\left(\nu \cdot \frac{\partial f\left(x_{1}, x_{2}\right)}{\partial x_{1}}\right)$ and $C=$ $\min _{\nu, x_{2}}\left(C_{1}\right)>0$. Then, for $p_{1}<\left(f_{1, \min }^{\prime}-\varepsilon\right)$, one has $\left|I_{\hbar, \nu}(p)\right|<\left|\mathcal{I}_{1}\left(x_{2}\right)\right|<e^{-C\left(f_{1, \min }^{\prime}-p_{1}-\varepsilon\right) / \hbar}$. Similarly, we define $f_{1, \max }^{\prime}, f_{2, \min }^{\prime}$ and $f_{2, \max }^{\prime}$ and obtain similar estimates. 


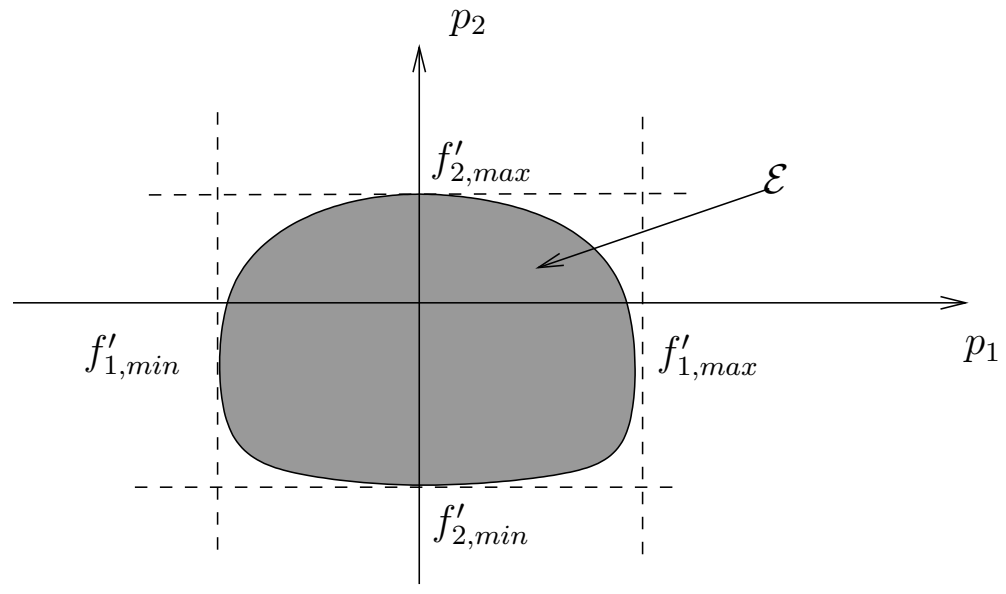

Figure 6: Picture of the domain $\mathcal{E}$

\section{Remark}

- We have shown that the integral $I_{\hbar, \nu}(p)$ is "small" outside a rectangle containing $p=0$ in the plane $\mathbb{R}^{2}$. This rectangle shrinks to 0 when the perturbation $f$ is $C_{1}$ small. Coming back to variables $\left(n^{\prime}, n\right)$, this means that for $n$ fixed, the matrix elements of $\left\langle n^{\prime}|\hat{M}| n\right\rangle$ are "small" except in a domain surrounding the point $n^{\prime}=M_{0}^{\mathfrak{t}} n$.

\subsection{Proof of Theorem 6}

Instead of the variables $n, n^{\prime} \in \mathbb{Z}^{2}$, we prefer to use for $n \neq 0$,

$$
h=\frac{1}{|n|}>0, \quad \nu=\frac{n}{|n|} \in S^{1}, \quad \nu^{\prime}=\frac{n^{\prime}}{|n|} \in \mathbb{R}^{2} .
$$

From (21), we have $p=\nu^{\prime}-M_{0}^{\mathrm{t}} \nu$. From (11) and (12), we have for $n \neq 0$ :

$$
R_{n^{\prime}, n} \stackrel{\text { def }}{=}\left\langle\hat{A} n^{\prime}|\hat{M}| \hat{A}^{-1} n\right\rangle=\exp \left(\frac{1}{h} A\left(\nu^{\prime}\right)\right)\left\langle n^{\prime}|\hat{M}| n\right\rangle
$$

with

$$
A\left(\nu^{\prime}\right)=a\left(\left|\nu_{u}\right|-\left|\nu_{s}\right|-\left|\nu_{u}^{\prime}\right|+\left|\nu_{s}^{\prime}\right|\right),
$$

where $n, h, \nu$ are considered as fixed in the discussion. Note that we do not use yet the notation $\left\langle n^{\prime}|\hat{R}| n\right\rangle$, but $R_{n^{\prime}, n}$ instead, because we don't know yet if $|n\rangle$ belongs to the domain of $\hat{R}$. This will be proved a few lines below.

Then Equation (22) gives the upper bound: $\left|\left\langle n^{\prime}|\hat{M}| n\right\rangle\right|<\exp \left(\frac{1}{h} B\left(\nu^{\prime}\right)\right)$, where the function $B\left(\nu^{\prime}\right)$ is equal to 0 on a rectangle domain denoted by $Z_{B}(\nu)$, containing the point $M_{0}^{\mathrm{t}} \nu$. The function $B\left(\nu^{\prime}\right)$ decreases linearly outside this rectangle, with a slope $2 \pi C$ which does not depend on $\nu$ and $h$, see Figure 7 . The size of the domain $Z_{B}(\nu)$ goes to 0 , whenever $\|f\|_{C^{1}} \rightarrow 0$. 
We deduce that $\left|R_{n^{\prime}, n}\right|<\exp \left(\frac{1}{h} F\left(\nu^{\prime}\right)\right)$, with $F\left(\nu^{\prime}\right)=A\left(\nu^{\prime}\right)+B\left(\nu^{\prime}\right)$. The function $A\left(\nu^{\prime}\right)$ is zero for $\nu^{\prime}=\nu$, and it is negative and decreases with a constant slope $a$ on a domain denoted by $Z_{A}(\nu)$, see Figure 7 . At the point $\nu^{\prime}=M_{0}^{\mathfrak{t}}(\nu)=\left(e^{\lambda_{0}} \nu_{u}, e^{-\lambda_{0}} \nu_{s}\right)$, the value of $A\left(\nu^{\prime}\right)=-a\left(\left|\nu_{u}\right|\left(e^{\lambda_{0}}-1\right)+\left|\nu_{s}\right|\left(1-e^{-\lambda_{0}}\right)\right)<\mathcal{A}<0$ is strictly negative, uniformly with respect to $\nu \in S^{1}$. Therefore, the domain $Z_{B}(\nu)$ is strictly included in $Z_{A}(\nu)$ if the perturbation $f$ is small enough in $C^{1}$ norm.

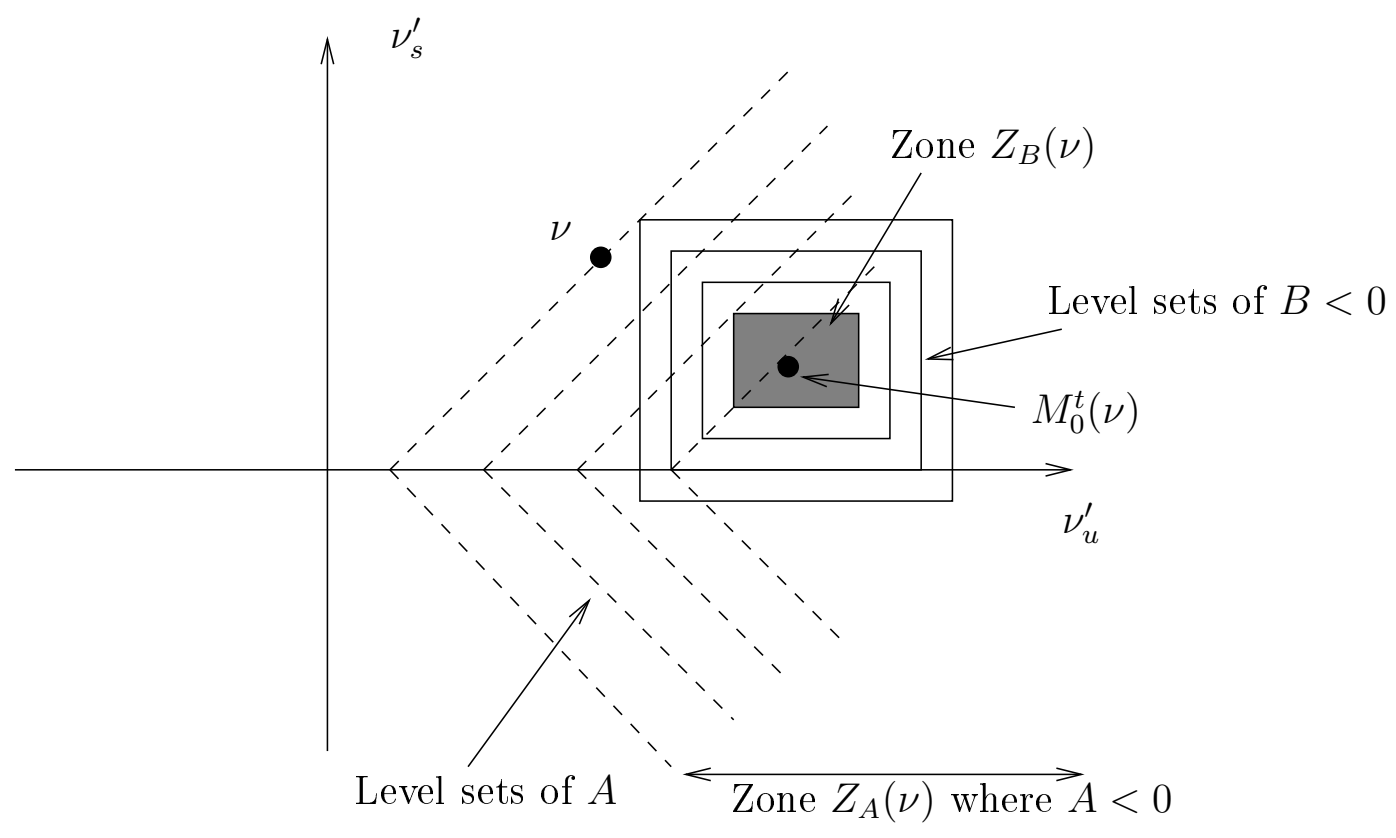

Figure 7: Schematic representation of level sets of functions $A\left(\nu^{\prime}\right)$ and $B\left(\nu^{\prime}\right)$, with respect to the frame of unstable/stable directions. Here $|\nu|=1$, and $\nu$ is sent to $M_{0}^{\mathrm{t}}(\nu)$ by the dynamics.

If we choose $a$ such that $A$ increases slower than $B$ decreases (i.e., $a<c^{\prime} 2 \pi C$ where $\left.c^{\prime}>0\right)$ then there exists $c>0$ such that

$$
F\left(\nu^{\prime}\right)<-c\left(\left|\nu_{1}\right|+\left|\nu_{2}\right|+\left|\nu_{1}^{\prime}\right|+\left|\nu_{2}^{\prime}\right|\right)
$$

This gives $\left|R_{n^{\prime}, n}\right|<\exp \left(-c\left(\left|n_{1}\right|+\left|n_{2}\right|+\left|n_{1}^{\prime}\right|+\left|n_{2}^{\prime}\right|\right)\right)$.

Let us first deduce that the operator $\hat{R}=\hat{A} \hat{M} \hat{A}^{-1}$ is defined on the domain $C_{A}$. If $|\phi\rangle \in C_{A}$,

$$
\begin{aligned}
|\phi\rangle & \in \operatorname{Dom}(\hat{R}) \Leftrightarrow \hat{M} \hat{A}^{-1}|\phi\rangle \in D_{A} \Leftrightarrow \sum_{n^{\prime}}\left|\left\langle\hat{A} n^{\prime}\left|\hat{M} \hat{A}^{-1}\right| \phi\right\rangle\right|^{2}<\infty \\
& \Leftrightarrow \sum_{n^{\prime}}\left|\sum_{n}\left\langle\hat{A} n^{\prime}|\hat{M}| \hat{A}^{-1} n\right\rangle\langle n \mid \phi\rangle\right|^{2}<\infty \Leftrightarrow \sum_{n^{\prime}}\left|\sum_{n} R_{n^{\prime}, n}\langle n \mid \phi\rangle\right|^{2}<\infty .
\end{aligned}
$$


Let us show now the last estimate is actually fulfilled. If $|\phi\rangle \in L^{2}\left(\mathbb{T}^{2}\right)$

$$
\begin{aligned}
\sum_{n^{\prime}}\left|\sum_{n} R_{n^{\prime}, n}\langle n \mid \phi\rangle\right|^{2} & \leq \sum_{n^{\prime}}\left(\sum_{n}\left|R_{n^{\prime}, n}\right||\langle n \mid \phi\rangle|\right)^{2} \\
\leq\|\phi\|^{2} & \sum_{n^{\prime}} e^{-2 c\left(\left|n_{1}^{\prime}\right|+\left|n_{2}^{\prime}\right|\right)}\left(\sum_{n} e^{-c\left(\left|n_{1}\right|+\left|n_{2}\right|\right)}\right)^{2}<\infty
\end{aligned}
$$

Therefore the operator $\hat{R}=\hat{A} \hat{M} \hat{A}^{-1}$ is defined on the domain $C_{A}$ and its matrix elements are obviously $\left\langle n^{\prime}|\hat{R}| n\right\rangle=R_{n^{\prime}, n}$. With the same arguments as those used earlier for the expanding map on $S^{1}$, we deduce that $\hat{R}$ extends to a trace class operator on $L^{2}\left(\mathbb{T}^{2}\right)$.

\subsubsection{Proof of the exponential concentration of R.P. resonances}

The proof of Theorem 7 is very similar to the proof we gave in Section 3.3. Here we use the same notations and emphasize the differences.

Let $\hat{R}$ be the trace class operator obtained in Theorem 6 , with the estimation $\left|\left\langle n^{\prime}|\hat{R}| n\right\rangle\right|<$ $\exp \left(-c\left(\left|n_{1}\right|+\left|n_{2}\right|+\left|n_{1}^{\prime}\right|+\left|n_{2}^{\prime}\right|\right)\right)$ on its matrix elements, with $c>0$.

Lemma 2. Let $\mu_{j}, j=0,1, \ldots$ be the non zero singular values of $\hat{R}$, such that $\mu_{j+1} \leq \mu_{j}$, repeated as many times as the value of their multiplicity. Then

$$
\mu_{j} \leq C_{1} e^{-\frac{c}{2} \sqrt{j}}
$$

with $C_{1}>0$.

Proof. From the min-max theorem,

$$
\mu_{j}=\min _{V \subset L^{2}\left(\mathbb{T}^{2}\right), \operatorname{codim} V=j} \max _{v \in V,\|v\|=1}\|\hat{R} v\| .
$$

Consider the Fourier basis $|n\rangle, n=\left(n_{1}, n_{2}\right) \in \mathbb{Z}^{2}$ and $V_{l}=\operatorname{span}(|n\rangle)_{\max \left(\left|n_{1}\right|,\left|n_{2}\right|\right)>l}$, hence $\operatorname{codim} V_{l}=(2 l+1)^{2}$. If $|v\rangle \in V_{l}$, we compute

$$
\begin{aligned}
\| \hat{R}|v\rangle \mid \| & =\| \sum_{n^{\prime} \in \mathbb{Z},|n|>l}\left|n^{\prime}\right\rangle\left\langle n^{\prime}|\hat{R}| n\right\rangle\langle n \mid v\rangle \| \\
& \leq\|v\|\left(\sum_{n^{\prime} \in \mathbb{Z}^{2}} \exp \left(-c\left(\left|n_{1}^{\prime}\right|+\left|n_{2}^{\prime}\right|\right)\right)\right)\left(\sum_{n / \max \left(\left|n_{1}\right|,\left|n_{2}\right|\right)>l} \exp \left(-c\left(\left|n_{1}\right|+\left|n_{2}\right|\right)\right)\right) \\
& \leq\|v\| C e^{-c l}, \quad C>0
\end{aligned}
$$

We deduce that $\mu_{j} \leq C_{1} e^{-c \sqrt{j} / 2}$, with $C_{1}>0$. 
For non zero eigenvalues, define

$$
l_{j}=\log \left|\lambda_{j}\right|, \quad m_{j}=\log \mu_{j}
$$

(These sequences tend to $-\infty$ as $j \rightarrow \infty$ ). Inequality (19) reads $\sum_{j=0}^{n} l_{j} \leq \sum_{j=0}^{n} m_{j}$. Eq. (23) gives $m_{j} \leq \log C_{1}-c \sqrt{j} / 2$. We deduce that $\sum_{j=0}^{n} l_{j} \leq(n+1) \log C_{1}-\frac{c}{2} \sum_{j=0}^{n} \sqrt{j}$. But $\sum_{j=0}^{n} \sqrt{j} \geq \int_{0}^{n} \sqrt{x} d x=\frac{2}{3} n^{3 / 2}$. Hence $\frac{1}{(n+1)} \sum_{j=0}^{n} l_{j} \leq \log C_{1}-\frac{c}{3} \frac{n^{3 / 2}}{(n+1)}$. But $l_{n} \leq l_{j}$ for $j \leq n$, so $l_{n} \leq \frac{1}{(n+1)} \sum_{j=0}^{n} l_{j} \leq \log C_{1}-\frac{c}{3} \frac{n^{1 / 2}}{(1+1 / n)}$, which proves Theorem 7 .

\section{Conclusion}

For specific models of chaotic dynamics, namely real analytic expanding maps on the circle $S^{1}$ and real analytic hyperbolic maps on the torus $\mathbb{T}^{2}$, we have shown that the decay of time correlation functions can be described by a trace class operator in $L^{2}\left(S^{1}\right)$ (resp. $L^{2}\left(\mathbb{T}^{2}\right)$ ). We have followed an approach similar to the "complex scaling method", well-known to study the decay of quantum states in open quantum systems. As explained in the introduction, this approach has been already used by V. Baladi and M Tsujii [4] for hyperbolic diffeomorphisms in a more general context, but our methods differ sightly and allowed us to obtain different results. To make a more precise comparison, our operator $\hat{A}$ defined in Eq.(11) and the conjugation in Eq.(12), correspond to their definition of anisotropic norms. But they use powers of the Fourier components whereas we use exponential of them. This exponential is important for us to obtain a trace class operator $\hat{R}$, as explained in section 3.2.1.

In this paper, some "semi-classical aspects" of hyperbolic dynamics have appeared many times: (i) in Theorem 10 concerning the localization of the matrix elements in Fourier space, and the remark which follows, (ii) in a remark on the semi-classical Weyl law after equation (13), and (iii) with Figure 5 where a cluster of eigenvalues suggests some semiclassical tunnelling effect. The role of semi-classical parameter is played by the inverse of the distance in Fourier space: $\hbar \equiv 1 /|n|$. A direction of research would be to make this semi-classical theory more precise.

We would like to comment some limitations of our results and possible extensions of them. First we have assumed that the dynamics is given by a real analytic map. This is a severe limitation because in hyperbolic dynamical system theory one has to use Hölder potential functions [3][14]. We have presented here the results in their simplest form. In particular, for expanding maps on the torus, we have shown that $\hat{R}$ is trace class for any expanding map, but for hyperbolic maps on the torus we have assumed that the non linear perturbation $f$ is weak enough. In geometric terms we have supposed that $\|f\|_{C^{1}}$ is weak enough so that the unstable and stable foliations are respectively contained in fixed cones (the cones adapted to the linear map, and which enter in the definition of $\hat{A}$ eq.(11)). It could be possible to generalize in this direction and treat in this way any uniform analytic hyperbolic map on the torus, using a local choice of cones. As in [4], this could be possible using pseudo-differential operators instead of $\hat{A}$. Then the localization property 
of the matrix elements in Theorem 10 would be replaced by a "microlocal" version in the cotangent space $T^{*} S^{1}$ (resp. $T^{*} \mathbb{T}^{2}$ ). Some other directions of research could be to take advantage of the relative simplicity of this approach to investigate non uniform hyperbolic dynamics or other kinds of dynamical systems which exhibit some chaotic behaviour, where many questions remain open.

\section{References}

[1] M. Abramowitz and I. A. Stegun. Handbook of mathematical functions. Dover, 1954.

[2] V.I. Arnold. Geometrical methods in the theory of ordinary differential equations. Springer Verlag, 1988.

[3] V. Baladi. Positive transfer operators and decay of correlations. Singapore: World Scientific, 2000.

[4] V. Baladi and M. Tsujii. Anisotropic Hölder and Sobolev spaces for hyperbolic diffeomorphisms. to appear in Ann. Inst. Fourier, 2005.

[5] M. Blank, G. Keller, and C. Liverani. Ruelle-Perronn-Frobenius spectrum for Anosov maps. Nonlinearity, 15:1905-1973, 2002.

[6] H.L. Cycon, R.G. Froese, W. Kirsch, and B. Simon. Schrödinger operators, with application to quantum mechanics and global geometry. (Springer Study ed.). Texts and Monographs in Physics. Berlin etc.: Springer-Verlag., 1987.

[7] J.P. Eckmann and D. Ruelle. Ergodic theory of chaos and strange attractors. Rev. Mod. Phys., 57(3):617-656, 1985.

[8] V. Baladi et L.-S. Young. On the spectra of randomly perturbed expanding maps. (Erratum Comm. Math. Phys., 166, 219-220 (1994)), Comm. Math. Phys. 156 (1993), 355-385.9.

[9] P. Gaspard. Chaos, scattering and statistical mechanics. Cambridge Nonlinear Science Series. 9. Cambridge: Cambridge University Press, 1998.

[10] I. Gohberg, S. Goldberg, and N. Krupnik. Traces and Determinants of Linear Operators. Birkhauser, 2000.

[11] S. Gouëzel and C. Liverani. Banach spaces adapted to Anosov systems. Ergodic Theory and dynamical systems, 26:189-217, 2005.

[12] L. Guillope, K. Lin, and M. Zworski. The Selberg zeta function for convex co-compact. Schottky groups. Comm. Math. Phys., 245(1):149-176, 2004.

[13] M. Gutzwiller. Chaos in classical and quantum mechanics. Springer-Verlag, 1991. 
[14] A. Katok and B. Hasselblatt. Introduction to the Modern Theory of Dynamical Systems. Cambridge University Press, 1995.

[15] C. Liverani. Fredholm determinants, anosov maps and ruelle resonances. Discrete and Continuous Dynamical Systems, 13(5):1203-1215, 2005.

[16] A. Martinez. An Introduction to Semiclassical and Microlocal Analysis. Universitext. New York, NY: Springer, 2002.

[17] S. Nonnenmacher. Spectral properties of noisy classical and quantum propagators. Nonlinearity, 16(5):1685-1713, 2003.

[18] M. Reed and B. Simon. Mathematical methods in physics, vol I : Functional Analysis. Academic press, New York, 1972.

[19] M. Reed and B. Simon. Mathematical methods in physics, vol IV : Analysis of operators. Academic Press, 1978.

[20] D. Ruelle. Locating resonances for axiom A dynamical systems. J. Stat. Phys., 44:281292, 1986.

[21] H. H. Rugh. The correlation spectrum for hyperbolic analytic maps. Nonlinearity, 5(6):1237-1263, 1992. 